\title{
MTGPLP Approach for Traffic Signal Intelligent Control
}

\author{
Ya Li, ${ }^{1,2}$ Renhuai Liu, ${ }^{1,2}$ Yuanyang Zou $\mathbb{D}^{3},{ }^{3}$ Yingshuang Ma, ${ }^{4}$ and Guoxin $\mathrm{Wang}^{5}$ \\ ${ }^{1}$ School of Management, Shanghai University, Shanghai 200444, China \\ ${ }^{2}$ School of Management, Hangzhou Dianzi University, Hangzhou 310018, China \\ ${ }^{3}$ School of Business Administration, Hubei University of Economics, Wuhan 430205, China \\ ${ }^{4}$ Business College, Huanggang Normal University, Huanggang, Hubei 438000, China \\ ${ }^{5}$ School of Mathematics and Physics, Nanyang Institute of Technology, Nanyang 473004, China \\ Correspondence should be addressed to Yuanyang Zou; yuanyzou@gmail.com
}

Received 7 May 2020; Revised 14 July 2020; Accepted 17 July 2020; Published 17 August 2020

Academic Editor: Alberto Cavallo

Copyright $\odot 2020 \mathrm{Ya} \mathrm{Li}$ et al. This is an open access article distributed under the Creative Commons Attribution License, which permits unrestricted use, distribution, and reproduction in any medium, provided the original work is properly cited.

In this paper, we state a combining programming approach to optimize traffic signal control problem. The objective of the model is to minimize the total queue length with weight factors at the end of each phase. Then, modified Twin Gaussian Process (MTGP) is employed to predict the arrival rates for the traffic signal control problem. For achieving automatic control of the traffic signal, an intelligent control method of the traffic signal is proposed in view of the combining method, that is to say, the combining method of MTGP and LP, called MTGPLP, is embraced in the intelligent control system. Furthermore, some numerical experiments are proposed to test the validity of the model and the MTGPLP approach. In particular, the results of numerical experiments show that the model is effective with different arrival rates, departure rates, and weight factors and the combining method is successful.

\section{Introduction}

The congest problem is a classic problem in the traffic control system. Specially, in the medium and large cities, the congestion phenomena are more intense at the intersections. That is to say, at the tail of the queue, a driver may remain a few cycles to traverse the intersection at peak periods. Therefore, there is an interesting question on how to make reasonable arrangement the green time at the intersection which is an issue aspect of the traffic control system in urban. In addition, there are several meanings that the sensible tactics are selected as follows: cut down congestion and degrade tail gas release of vehicles [1]. Thus, this study is to investigate the signal at the intersection.

Many sophisticated methods were stated to cope with the traffic signal problem over the past few decades. The studies can be summarized into three classifications: fixed cycle time systems, semifixed systems, and intelligent systems.

The characteristic of the fixed systems is to build the signal scheduling based on the periodic prearranged time. The first comprehensive study of the traffic signal control was introduced by Webster [2]. Afterwards, Robertson [3] stated a technique to cope with the traffic light length problem at the intersection, called TRANSYT. Cantarella and Improta [4] constructed a multiple objectives system which was to maximize the volume factor and to minimize the periodic time to cope with traffic signal problem. In the sequel, the minimization of several criteria (e.g., mean delay and average number of stops) that were the network of signalised junctions was considered by Reljic [5] who utilized a dynamic multicultural programming approach to tackle the problems. In the next year, a parallel computing approach that was used to solve the traffic signal problem was introduced by Wong [6]. An optimization technique was stated based on the entrance lane by Wong et al. [7]. Because the fixed system is nonapplication with the development of computer technology, a few studies for the traffic signal problem were studied by this method in the last decades.

The characteristic of the semifixed models is to construct segmentally varying foremost traffic light length scheduling for traffic intersection. For this kind of method, the Sydney 
coordinated adaptive traffic systems, which have been broadly utilized to compute the division time, offsets, and periodic time being in line with present traffic light cycle length prerequisite, was proposed by Lowrie [8] firstly. The split cycle offset optimization technique (SCOOT) was utilized to deal with the traffic signal control problems by Bretherton and Browen [9], Bretherton [10], and Sharma and Gidde [11]. The likelihood methods that were stated by Wey [12] and Clempner and Poznyak [13] were employed to cope with the traffic light planning problems. Real-time semifixed traffic light scheduling systems, which were constructed on the real time, were introduced by Head et al. [14]; Mirchandani and Head [15] introduced and utilized the system to optimize phase time and delay for the traffic signal problem. A discrete time system for optimizing the traffic signal control problem was introduced by Dotoli et al. [16]. The phase time and delay time were studied by multiobjective signal control systems based on a fuzzy logic approach were addressed by Schmöcker et al. [17]. Moreover, the mixed integer linear programming systems that contain 0-1 variables, which minimize the cycle length at the junction, were presented by Wong and Wong $[7,18]$ and Wong et al. [19]. Based on the data-driven method, some semi-intelligent systems were presented by Zhang et al. [20].

In addition, the intelligent systems have drawn attention. For dealing with the multimodal urban traffic signal systems, a predictive model, which included two objectives, was studied by Kachroudi and Bhouri [21]. Multiple objective systems for solving the traffic light length problems at the intersection were depicted by Cai et al. [22], Khamis and Gomaa [23], and Dujardin et al. [24]. The goal of the former was to optimize active assignment of green light length, adjust the regulator arguments automatically, and revise the traffic light plans speedily. A multiagent structure was constructed to handle with the traffic light length problems at the intersection of the latter study. Furthermore, some intelligence approaches for coping with the multiple objective systems for traffic light control problems were introduced by Garcia-Nieto et al. [25] and Li et al. [26]. At the same year, Coll et al. [27] built a single-objective model to deal with the traffic light length problem at the junction. In the light of the research of Dujardin et al. [24], two classes' intelligent systems are prevalent in the current study due to the relay on the gen about circumstances provided regularly by some monitor equipment. Furthermore, Friesz et al. [28] and Liu and Smith [29] stated approaches to cope with traffic signal control problems in which the models contained 0-1 variables for controlling the traffic light. Notwithstanding, continuum traffic light signalised intersection systems that can prevent the noncontinuous variable into optimizing the junction signal time were introduced by Han et al. [30], Han and Gayah [31], and Han et al. [32]. One cell automata traffic stream system that investigates the three phases' concept was stated by Qian et al. [33]; the paper gave four verdicts in the light of the numerical experiments. In the same year, a mixed traffic stream cell automaton system with the influence of speeding up the entrance lane to handle with the junction signal programming problem was described by Qian, et al. [34]; the study studied the driving characteristics of cars following station on the inflow lanes at the intersections. Moreover, a single junction model, which embraces vehicles and pedestrian, was introduced by Yu et al. [35]. In addition, some control systems for optimizing traffic signal based on adaptive approaches were proposed. Manolis et al. [36] constructed a management and control system (MCS) to handle with the traffic signal and gave a novel adaptive method called Adaptive Fine-Tuning (AFT) in the study. An adaptive Kalman filter (AKF) model was employed to predict accumulative travel time for each phase in the traveltime responsive approach to be introduced by Choi et al. [37] in the same year. For dealing with the traffic signal, Q-Learning approaches were applied to solve the traffic control [38-42]. In this paper, an intelligent traffic light control system is also stated with single junctions, which is part of the intelligent systems. At last, in the past 20 years, machine learning is an exceedingly region no matter in theory or in engineering applications. The machine learning methods for dealing with the traffic signal control problems also were comprehensively pondered [43]. For optimizing the traffic signal at a single intersection, neural networks methods and convolution neural networks approaches were utilized to optimize the traffic signal plans [44-47]. In addition, deep reinforcement learning techniques were applied to solve the traffic signal schemes [48-51]. Furthermore, the optimum traffic signal timing plans were governed with Q-Learning [52-56].

Furthermore, note that some similar works (eg., Ribeiro and Simões [57]; Simões and Ribeiro [58]; Simões and Ribeiro [59]; and Simões and Ribeiro [60] studied the urban traffic light control and, however, some complementarity constraints are contained in the systems, which are very hard to solve because of their exceeding nonconvexity and combinatorial construction. In this paper, some ways are utilized to overcome the $0-1$ variables in the model.

In addition, a similar linear programming for the traffic signal problem was studied by Liu et al. [61]. In order to calculate the departure flows, a parameter set should be given firstly. Furthermore, a sufficient global optimality condition and the efficiency of the model for Liu et al. [61] was presented by Li et al. [62]. A linear programming model for oversaturated situation of the intersection signal time was considered by Zhao et al. [63] who proposed a fast greedy search algorithm to solve the model. A stochastic linear programming model that was employed to solve the oversaturated intersections problem was stated by Tong et al. [64]. In the similarity studies, the arrival rates are assumed to obey Poisson distribution or the average value of the latest several cycles. The arrival rates for each phase are different in general. On the contrary, the linear model was also studied by Zou and $\mathrm{Hu}$ [65] and Zou et al. [66]. In the former study, the complementary constraints of the model were embraced and it was a nonconvex problem; thus, a model by nonlinear constraints was employed to approximate the nonconvex model. In the latter investigation, the NLRMNSGA-II contained a prediction algorithm, but the algorithm was a single output approach. Thus, we give a new approach which is a multiple input and multiple output algorithm to predict the inflow arrival rates since every cycle contains four 
phases. That is, a linear model with linear constraints and a multioutput algorithm is stated.

Compared to the previous work, these are two works in this paper:

(i) A modified multi-input and multioutput algorithm regression algorithm, called modified Twin Gaussian Process (MTGP), is presented, which is based on the Twin Gaussian Process [67].

(ii) In addition, a combination approach, called MTGPLP, is introduced to cope with the model, which combines the MTGP approach and linear programming method. In particular, the arrival rates are estimated by the MTGP method based on the number of arrival vehicles counted by monitors at each cycle in the latest several cycles or at the same time of the former several days.

The remainder of this paper is organized as follows. In Section 2, the characteristic of the intersection is illustrated and the traffic intersection signal control model is formulated as an linear program model with linear complementarity constraints. The algorithm is shown in Section 3. In Section 4, numerical experiments are shown. Lastly, the conclusion is arranged in Section 5.

\section{Coordinated Signal Control Problem and Problem Formulation}

The 4-phase traffic light control system is investigated in this paper (e.g., Figure 1). In Figure 1, there are 8 inflow lanes to be considered. Therefore, the light cycle can be stated as Figure 2. From Figure 2, the traffic lights have three states: green, amber, and red.

Let $j=1,2,3,4$ stand for the index of the phases of each cycle and $k+1(k=0,1, \ldots, N-1)$ denote the index of the cycles (Figure 2 ); $N$ is the total cycles; $i=1, \ldots, 8$ denotes the index of inflow lanes; $y, d_{y}$, and $d_{c}$, respectively, are the green time, amber time, and clearance time. According to the conservation law of the phase light time, a cycle length can be articulated as

$$
t_{k+1}-t_{k}=y_{1, k+1}+y_{2, k+1}+y_{3, k+1}+y_{4, k+1}+4\left(d_{y}+d_{c}\right),
$$

and the vehicles queue length can be formulated as

$$
\begin{aligned}
x_{i, l, k+1} & =\max \left\{\max \left\{x_{i, l-1, k+1}, 0\right\}+A_{i, l, k+1}-D_{i, l, k}, 0\right\} \\
& =\max \left\{x_{i, l-1, k+1}+A_{i, l, k+1}-D_{i, l, k}, A_{i, l, k+1}-D_{i, l, k}, 0\right\},
\end{aligned}
$$

where $x_{i, l, k+1}$ and $x_{i, l-1, k+1}$, respectively, denote the vehicles' queue length in inflow lane $i$ at the end of phase $l$ and phase $l-1$ during the signal light cycle $k+1 ; A_{i, l, k+1}$ and $D_{i, l, k+1}$, respectively, stand for the entrance vehicles' length and departure vehicles' queue in inflow lane $i$ at the end of phase $l$ during the cycle $k$; $\max \{a, b\}$ means the bigger value between $a$ and $b$; and $x_{i, 4, k}:=x_{i, 0, k+1}$.
Let $\lambda_{i, l, k+1} \geq 0$ and $\mu_{i, l, k+1} \geq 0$, respectively, are the arrival rate and departure rate of inflow lane $i$ in phase $l$ during cycle $k+1$. Then, combining equations (1) and (2), the queue length in entrance lane $i$ at the end of phase $l$ during cycle $k+1$ can be reformulated as follows:

$$
\begin{aligned}
x_{i, l, k+1}= & \max \left\{\max \left\{x_{i, l, k-1}, 0\right\}+\lambda_{i, l, k+1}\left(y_{l, k+1}+d_{y}\right)\right. \\
& \left.-\mu_{i, k+1}\left(y_{l, k+1}+d_{y}\right) \delta_{l, k+1}, 0\right\} \\
& +\lambda_{i, l, k+1} d_{C},
\end{aligned}
$$

where

$\delta_{l, k+1}= \begin{cases}1, & \text { if green light of inflow lane } i \text { inphase } l \text { duringcycle } k+1, \\ 0, & \text { otherwise, }\end{cases}$

is a $0-1$ variable.

Let $\mathbf{x}_{l, k+1}=\left[x_{1, l, k+1}, \ldots, x_{8, l, k+1}\right]^{T}, \mathbf{A}_{l, k+1}=\left[A_{1, l, k+1}, \ldots\right.$, $\left.A_{8, l, k+1}\right]^{T}$, and $\mathbf{D}_{l, k}=\left[D_{8, l, k}, \ldots, D_{8, l, k}\right]^{T}$. Equation (2) can be rewritten as

$$
\mathbf{x}_{l, k+1}=\max \left\{\max \left\{\mathbf{x}_{l-1, k+1}, \mathbf{0}\right\}+\mathbf{A}_{l, k+1}-\mathbf{D}_{l, k}, \mathbf{0}\right\},
$$

where $\mathbf{0}$ is the zero vector and $\max \{\mathbf{a}, \mathbf{b}\}$ denotes the bigger value elements between $a_{i}$ and $b_{i}(i=1, \ldots, 8)$ in this paper.

Furthermore, let $\Lambda_{l, k+1}=\left[\lambda_{1, l, k+1}, \ldots, \lambda_{8, l, k+1}\right]^{T}$ and $\Upsilon_{l, k+1}=\left[\mu_{1, l, k+1}, \ldots, \mu_{8, l, k+1}\right]^{T}$; equation (4) can be reformulated as

$$
\begin{aligned}
\mathbf{x}_{l, k+1}= & \max \left\{\max \left\{\mathbf{x}_{l-1, k+1}, \mathbf{0}\right\}+\boldsymbol{\Lambda}_{l, k+1}\left(y_{l, k+1}+d_{y}\right)\right. \\
& \left.-\Upsilon_{l, k+1}\left(y_{l, k+1}+d_{y}\right) \delta_{l, k+1}, \mathbf{0}\right\}+\boldsymbol{\Lambda}_{l, k+1} d_{C},
\end{aligned}
$$

where $\mathbf{x}_{4, k}:=\mathbf{x}_{0, k+1}$.

Suppose that the queue lengths of all inflow lanes are equal to zero at the beginning of the first phase in the first cycle; then, the arrival rates are not less than the departure rates during the first phase in the first cycle. Thus, the queue length $x_{i, 1,1} \geq 0(i=1, \ldots, 8)$ at the end of the first phase in the first cycle, that is, $\mathbf{x}_{1,1} \geq \mathbf{0}$. Then, equation (6) can be rewritten as

$$
\begin{aligned}
\mathbf{x}_{l, k+1}= & \max \left\{\mathbf{x}_{l-1, k+1}+\Lambda_{l, k+1}\left(y_{l, k+1}+d_{y}\right)\right. \\
& \left.-\Upsilon_{l, k+1}\left(y_{l, k+1}+d_{y}\right) \delta_{l, k+1}, \mathbf{0}\right\}+\Lambda_{l, k+1} d_{C} \\
= & \max \left\{\mathbf{x}_{l-1, k+1}+\Lambda_{l, k+1}\left(y_{l, k+1}+d_{y}\right)\right. \\
& \left.-\Upsilon_{l, k+1}\left(y_{l, k+1}+d_{y}\right) \delta_{l, k+1}+\Lambda_{l, k+1} d_{C}, \Lambda_{l, k+1} d_{C}\right\} .
\end{aligned}
$$

In addition, let $\mathbf{I}_{1}=[-1,-1,0,0,0,0,0,0]^{T}$, $\mathbf{I}_{2}=[0,0,-1,-1,0,0,0,0]^{T}, \quad \mathbf{I}_{3}=[0,0,0,0,-1,-1,0,0]^{T}$, and $\mathbf{I}_{4}=[0,0,0,0,0,0,-1,-1]^{T}$. Then, equation (6) can be rewritten as

$$
\begin{aligned}
\mathbf{x}_{l, k+1}= & \max \left\{\mathbf{x}_{l-1, k+1}+\Lambda_{l, k+1}\left(y_{l, k+1}+d_{y}\right)\right. \\
& \left.-\Upsilon_{l, k+1}\left(y_{l, k+1}+d_{y}\right) \circ \mathbf{I}_{1}+\Lambda_{l, \mathrm{k}+1} \mathrm{~d}_{\mathrm{C}}, \Lambda_{l, \mathrm{k}+1} \mathrm{~d}_{\mathrm{C}}\right\} .
\end{aligned}
$$

Here, the operator "o" denotes the Hadamard product. 


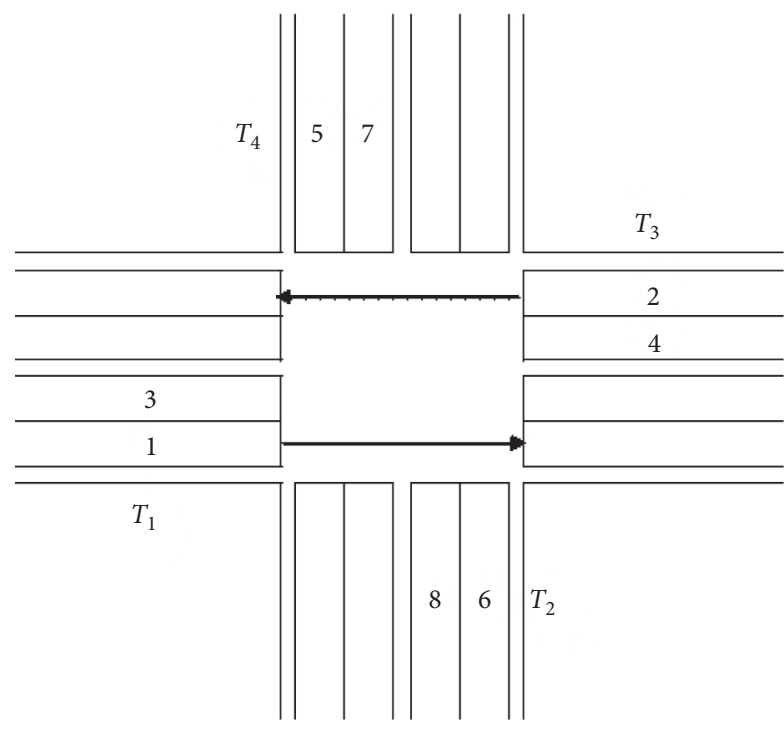

(a)

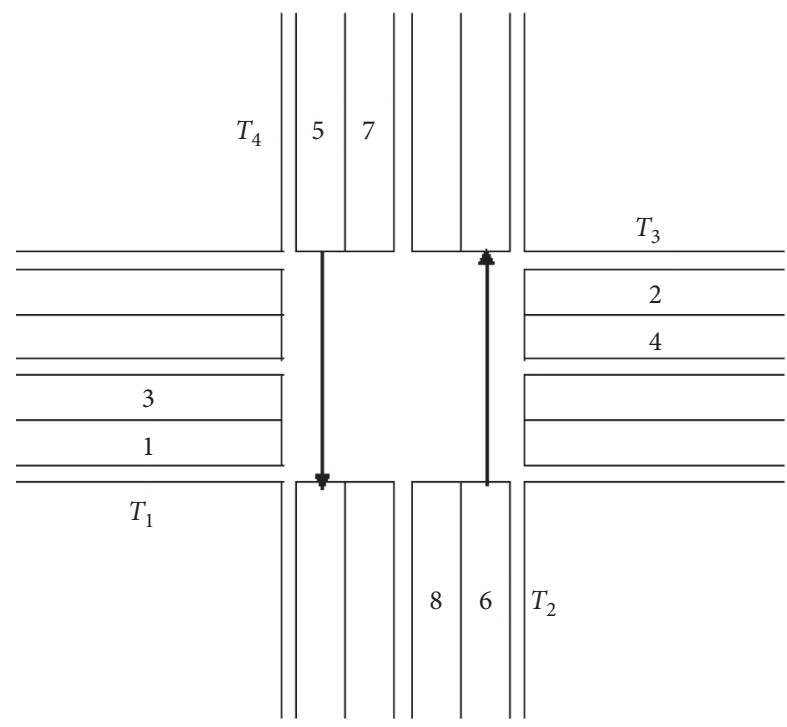

(c)

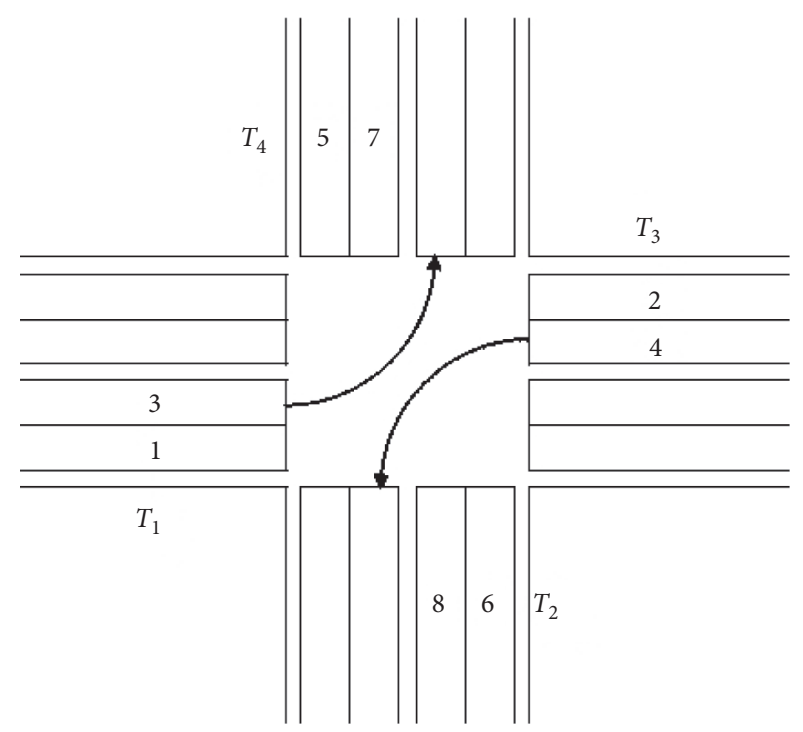

(b)

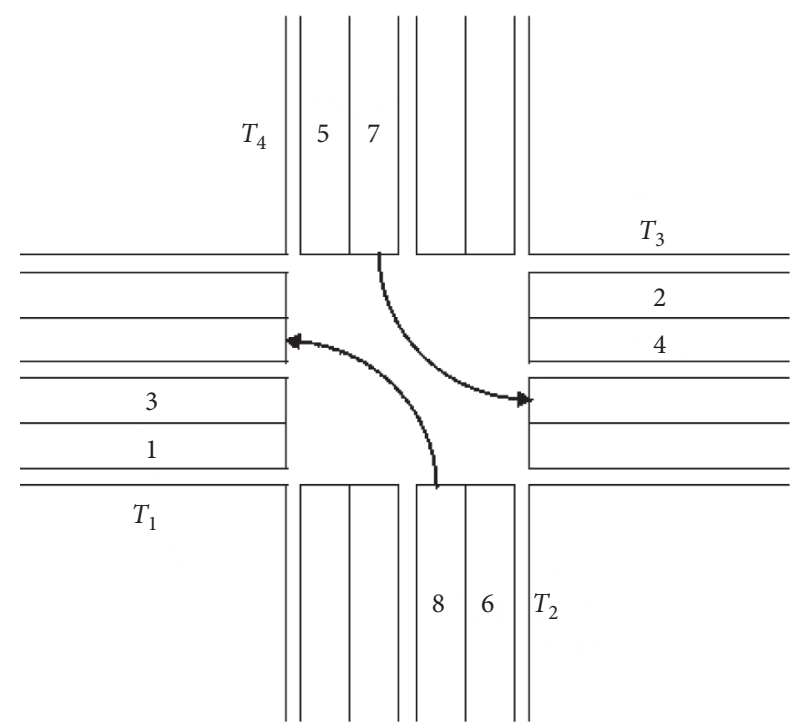

(d)

Figure 1: A typical 4-phase signal with left-turn protection control. (a) Phase 1. (b) Phase 2. (c) Phase 3. (d) Phase 4.

\begin{tabular}{|c|c|c|c|c|c|c|c|c|c|c|c|c|}
\hline \multirow{4}{*}{$\begin{array}{l}\text { Lanes } 1 \text { and } 2 \\
\text { Lanes } 3 \text { and } 4 \\
\text { Lanes } 5 \text { and } 6 \\
\text { Lanes } 7 \text { and } 8\end{array}$} & Green & Yellow & Red & \multicolumn{9}{|c|}{ Red } \\
\hline & \multicolumn{3}{|c|}{ Red } & Green & Yellow & Red & \multicolumn{6}{|c|}{ Red } \\
\hline & \multicolumn{6}{|c|}{ Red } & Green & Yellow & Red & \multicolumn{3}{|c|}{ Red } \\
\hline & \multicolumn{9}{|c|}{ Red } & Green & | Yellow & Red \\
\hline
\end{tabular}

Figure 2: Diagram of signal timing.

In what follows, we consider the following optimization problem:

$$
\min \sum_{k=0}^{N-1} \sum_{l=1}^{4} \gamma_{l, k+1}^{T} \mathbf{x}_{l, k+1}
$$

where $\gamma_{l, k+1}=\left[\gamma_{1, l, k+1}, \gamma_{2, l, k+1}, \ldots, \gamma_{8, l, k+1}\right]^{T}$ is the wight factor vector. Equation (9) is the objective of the study to minimize the sum of the queue length at the end of phase $l$ of all entrance lanes in cycle $k+1$ with weight factors; $x_{i, l, k+1}$ is expressed as (3); the green time $y_{i, l, k} \in[m, u], \quad k=0, \ldots, N-1$, $l=1,2,3,4, i=1,2 \ldots, 8$; and $m$ and $u$ are the lower bound and upper bound of the green time length, respectively.

In model (9), another question is how to determine the weight of each entrance lane. In general, the residential area and the working space are different (Figure 3). Because the 


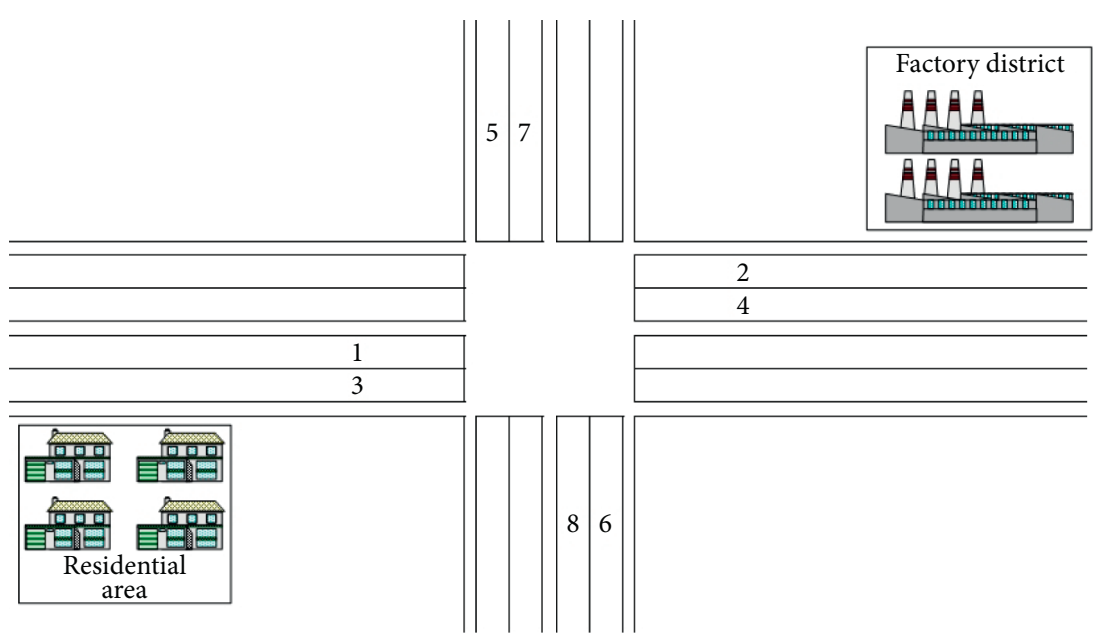

FIGURE 3: Diagram of signal timing.

workers go to work in the morning, thus, the weight factors of entrance lanes 1 and 3 are bigger than entrance lanes 2, 4, $5,6,7$, and 8 . On the contrary, when the workers go home in the afternoon, the weight factors of entrance lanes 2 and 4 are bigger than entrance lanes $1,3,5,6,7$, and 8 . Thus, the weight factors in model (9) can be calculated by statistical methods.

Then, combining equations (8) and (9), an optimizing system of the traffic light control is formulated as

$$
\begin{array}{ll}
\min & \sum_{k=0}^{N-1} \sum_{l=1}^{4} \gamma_{l, k+1}^{T} \mathbf{x}_{l, k+1}, \\
\text { s.t. } & \mathbf{x}_{l-1, k+1}+\boldsymbol{\Lambda}_{l, k+1}\left(y_{l, k+1}+d_{y}\right)-\Upsilon_{l, k+1}\left(y_{l, k+1}+d_{y}\right) \circ \mathbf{I}_{l} \\
& +\boldsymbol{\Lambda}_{l, k+1} d_{C}-\mathbf{x}_{l, k+1} \leq \mathbf{0}, \\
& \boldsymbol{\Lambda}_{l, k+1} d_{C}-\mathbf{x}_{l, k+1} \leq \mathbf{0}, \\
& m_{l, k+1} \leq y_{l, k} \leq u_{l, k}, \\
& \quad k=0, \ldots, N-1, l=1,2,3,4 .
\end{array}
$$

Furthermore, problem (10) can be rewritten as

$$
\begin{array}{ll}
\min & \Gamma^{T} \mathbf{x}, \\
\text { s.t. } & {\left[\begin{array}{cc}
\mathbf{B} & \mathbf{C} \\
-\mathbf{E} & \mathbf{0}
\end{array}\right]\left[\begin{array}{l}
\mathbf{x} \\
\mathbf{y}
\end{array}\right]+\left[\begin{array}{l}
\mathbf{p} \\
\mathbf{q}
\end{array}\right] \leq \mathbf{0},} \\
& \mathbf{m} \leq \mathbf{y} \leq \mathbf{u} .
\end{array}
$$

In view of model (11), the signal problem can be transferred a linear programming.

\section{MTGPLP Algorithm}

Note that many publications such as Ribeiro and Simões [57]; Simões and Ribeiro [58]; Simões and Ribeiro [59]; and Ribeiro and Simões [60] assumed that the arrival rates obey Poisson distribution. The memoryless is one of the characteristics of Poisson distribution, that is, the arrival rates of the cycle $k$ are never influenced to the former several cycles.
Thus, the assumption is improper. In addition, the mean value of former several cycles' arrival rates is set to the current cycle arrival rates [68]. That is, the arrival rate was a constant. However, it is not sensible in reality. These shortages are surmounted in the study.

The scarcity of the former situation is obvious. Comparing with the former method, the latter is more reasonable. However, the arrival rate is defined as the mean of the former several cycles' arrival rates, which does not consider the trend changing of the arrival rate. To illustrate this point, an example is listed. In Figure 4, the square points stand for the arrival rates which are predicted by the nonlinear regression method; the circle points denote the real arrival rates, and the rhombus points are the arrival rates which are equal to the average value of the former six phases' arrival rates. From Figure 4, we can obtain the following conclusions. If the arrival rates have a small change, the arrival rate estimated by the NLR method is approximately equal to the average value of the former several arrival rates. While the arrival rates change widely, the arrival rate estimated by the NLR method is more approximate to the real arrival rate than the average value method. That is, the NLR is an extremely sensible method to be employed to estimate the arrival rate at the intersection. In addition, four phases are considered of each cycle in the study. In other words, four sets of the arrival rates are needed to be predicted. Thus, a modified algorithm, called MTGP, is presented, which is based on a multioutput algorithm Twin Gaussian Process (TGP) [67] and employed to predict the arrival rates in every cycle. Note also that, in these studies, the arrival rates were set for all cycles, whereas, in this paper, the arrival rates are considered for all phases and the arrival rates are predicted by MTGP, which means, the values of the arrival rates are more reasonable in this study.

In the training step of TGP algorithm, the kernel is expressed as follows:

$$
\text { kernel }=K\left(\Lambda_{r}, \Lambda_{r}\right) \text {, }
$$

where $X_{r}$ is the input value of the training dataset. And 


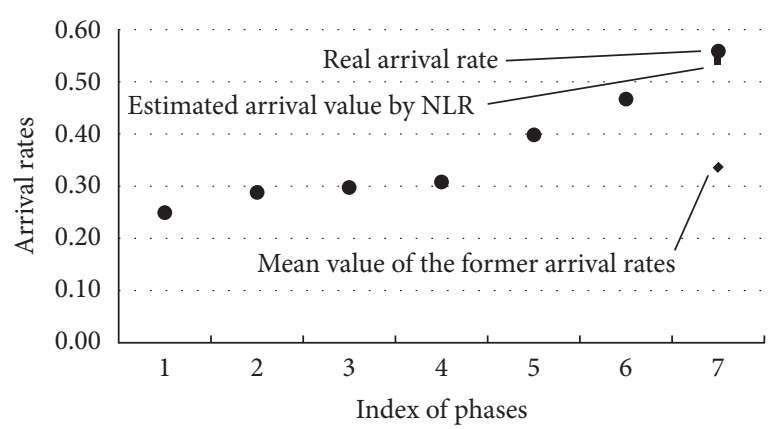

(a)

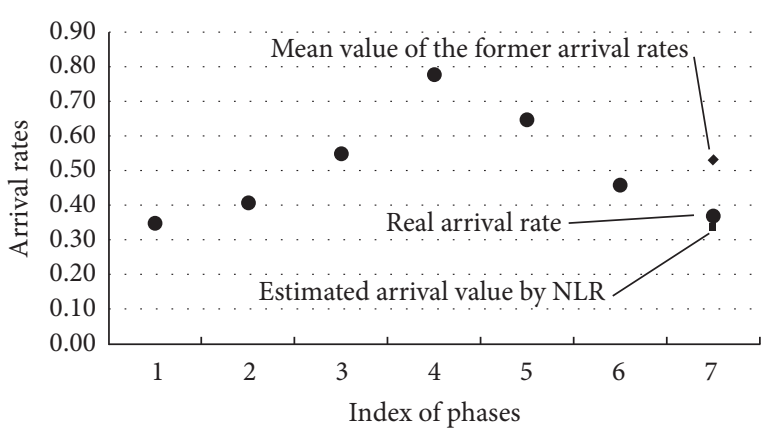

(b)

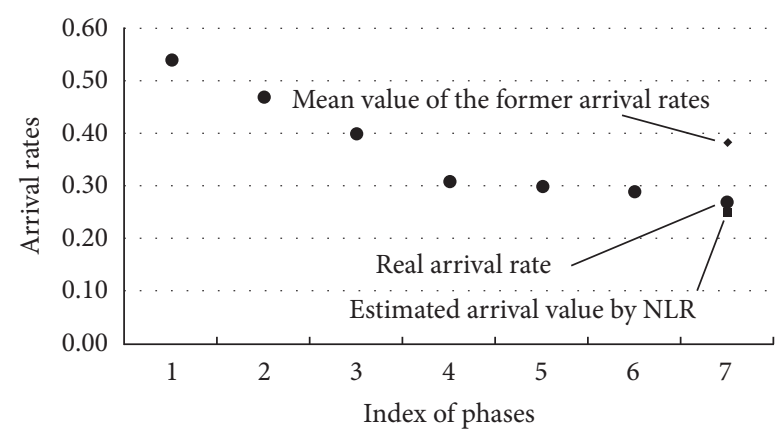

(c)

FIgURE 4: Example of the estimated arrival rate. (a) Arrival rates increase. (b) Arrival rates first increase and then decrease. (c) Arrival rates decrease.

$$
\text { kernel }=K\left(\Lambda_{r}, \Lambda_{r}\right)=\exp \left(-\gamma_{\Lambda_{r}}\left\|\lambda_{i}, \lambda_{j}\right\|^{2}\right)+\rho_{\Lambda_{r}} \delta_{i j}
$$

is defined by Bo and Sminchisescu [67], where $\lambda_{i}, \lambda_{j} \in \Lambda_{r}$ are the training data. In this study, the $\Lambda_{r}$ denotes the input of the arrival rates of training dataset, $\rho_{\Lambda_{r}}$ is a regularization parameter, and $\delta_{i j}$ is an indicator function.

When the training process is executed, the input testing dataset is not considered in the kernel (equation (12)). However, the input training dataset and input test dataset are from the same dataset. In fact, the input training data and input test data are relative. Thus, in view of equation (12), a new kernel is presented as follows:

$$
m \text { kernel }=K\left(X_{r}, X_{t}\right) \text {, }
$$

where $\Lambda_{t}$ is the input of the testing dataset. In this paper, $\Lambda_{t}$ denotes the input of arrival rates' dataset of testing dataset. The modified kernel of MTGP is stated as

$$
\begin{aligned}
\text { mkernel }= & K\left(\Lambda_{r}, \Lambda_{t}\right)=\exp \left(-\gamma_{\Lambda_{r}}\left\|\lambda_{i}, \lambda_{j}\right\|^{2}-\left\|\lambda_{i}, \lambda_{l}\right\|^{2}\right) \\
& +\rho_{\Lambda_{r}} \delta_{i j}+\rho_{\Lambda_{r t}} \delta_{i l}, \\
\text { or } m \text { kernel }= & K\left(\Lambda_{r}, \Lambda_{t}\right)=\alpha K\left(\Lambda_{r}, \Lambda_{r}\right)+(1-\alpha) K\left(\Lambda_{r}, \Lambda_{t}\right) \\
= & \alpha\left(\exp \left(-\gamma_{\Lambda_{r}}\left\|\lambda_{i}, \lambda_{j}\right\|^{2}\right)+\rho_{\Lambda_{r}} \delta_{i j}+(1-\alpha)\right) \\
& \cdot\left(\exp \left(-\left\|\lambda_{i}, \lambda_{l}\right\|^{2}\right)+\rho_{\Lambda_{r t}} \delta_{i l}\right),
\end{aligned}
$$

where $\alpha \in[0,1]$ is a parameter, $\lambda_{l}$ in $\lambda_{t}$ is the input of the arrival rates of testing data; and $\rho_{\Lambda_{r}}, \rho_{\Lambda_{r t}}$ are the parameters. In this paper, $\Lambda_{r}$ and $\Lambda_{t}$ are the arrival rates' datasets.

In what follows, the MTGPLP algorithm to solve system (11) is presented:

(i) Step 0 (initialization): set MTGPLP parameters; $\varepsilon_{0}, k=0, \lambda_{0}$; tolerances: $\varepsilon_{\min }, k_{\max }$

(ii) Step 1: calculate the kernel with training dataset and test dataset by equation (16)

(iii) Step 2: calculate the predicted values with the test dataset by equation (19) in Bo and Sminchisescu [67]

(iv) Step 3: solve the linear programming problem (11) by LP algorithm

Then, an intelligent control framework is presented as Figure 5. The monitors are employed to count the number of vehicles of each phase and every lane and to transfer the data to the processor. The processor is utilized to predict the arrival rates of the next cycle by MTGPLP and to assign the signal plan to the signal controller. The signal controller is used to distribute the green length for lights. Combining the algorithm, in this investigation, if the historical datasets of the arrival rates were memorized, the MTGP can be utilized to estimate the arrival rates adaptively.

Generally speaking, the intelligent control system of the traffic signal at intersection can be briefly summarized as three parts:

(i) Record the numbers of arrival vehicles and departure vehicles by the monitors in the latest $k-n$ cycles 


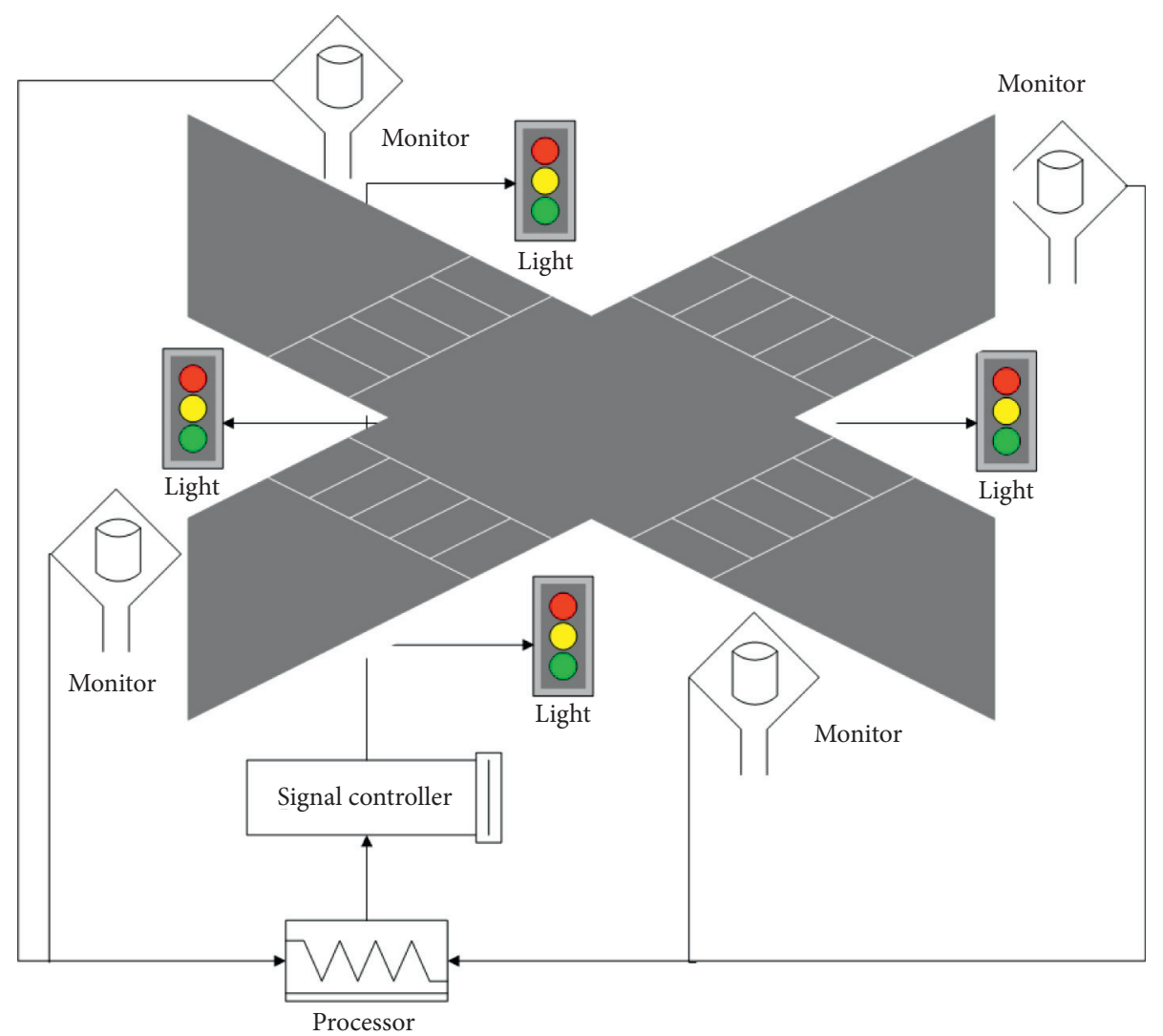

FIGURE 5: Intelligent control framework.

(ii) Utilize the MTGPLP algorithm to predict the arrival rate $\bar{\lambda}$ of each phase at cycle $k+1$ and calculate the green time of each phase of cycle $k+1$

(iii) Put the green time data into the control equipment of the traffic light

Then, in Section 4, the validity of the MTGP approach is tested firstly, immediately after some numerical experiments are employed to verify the validity of system (11) and the MTGPLP algorithm.

\section{Numerical Experiments}

The first task is to check the MTGP algorithm. That is, if the MTGP is worse than TGP, the MTGP is insignificance and not selected to predict the arrival rates. Because the MTGP is based on TGP and TGP was introduced by Bo and Sminchisescu [67], the HumanEva-I dataset [69] of the Bo and Sminchisescu [67] are utilized to test the MTGP. In the MTGP algorithm, the kernel is defined as equation (16) and $\alpha=0.9$. The results are shown in Figure 6 .

Since TMGP algorithm is generated by TGP, the same datasets are utilized to test the effectiveness of MTGP. " $S_{1}$ " " $S_{2}$," and " $S_{3}$ " denote the subjects in the experiment and "Box," "Gesture," "Jog," and "Walking" stand for the actions of the experimental subjects. That is, in HumanEva-I, " $S_{1}$," " $S_{2}$," and " $S_{3}$ " are three people, who do the box, gesture, jog, and walking actions, and the data of the actions are recorded by videos and cameras. "AME" means absolute mean error

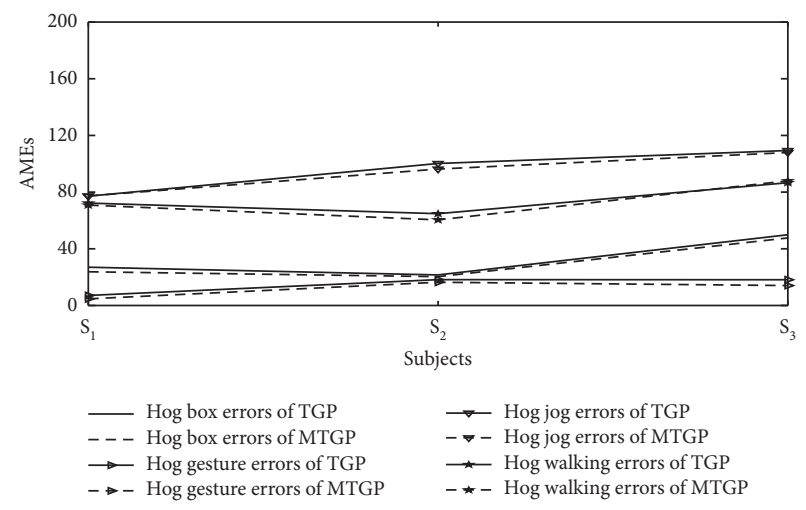

Figure 6: AMEs of TGP and MTGP.

of the predicted outputs and test outputs and is defined as follows:

$$
\mathrm{AME}=\operatorname{mean}(\text { abs }(\text { Predicts }- \text { TestTargets })) .
$$

From Figure 6, the mean absolute errors of the "Box" of " $S_{1}$," " $S_{2}$," and " $S_{3}$ " that are calculated by algorithm TGP are equal to $27.00,21.55$, and 49.91 , respectively. The absolute mean errors of the "Box" of " $S_{1}$," " $S_{2}$ ", and " $S_{3}$ " that are calculated MTGP are 23.78, 20.35, and 47.66, respectively. The mean absolute errors of the "Gesture" of " $S_{1}$," " $S_{2}$," and " $S_{3}$ " that are calculated algorithm TGP are 7.08, 18.23, and 18.16, respectively. The mean absolute errors of the 
"Gesture" of " $S_{1}$," " $S_{2}$," and " $S_{3}$ " that are calculated MTGP are $4.6678,16.3993$, and 14.0668 , respectively. The mean absolute errors of the "Jog" of " $S_{1}$ ", " $S_{2}$," and " $S_{3}$ " that are calculated algorithm TGP are 77.13, 100.21, and 109.36, respectively. The mean absolute errors of the "Jog" of " $S_{1}$," " $S_{2}$," and " $S_{3}$ " that are calculated MTGP are 77.29, 96.27, and 107.94, respectively. The mean absolute errors of the "Walking" of " $S_{1}$," " $S_{2}$ ", and " $S_{3}$ " that are calculated algorithm TGP are 72.26, 64.78, and 86.64, respectively. The absolute mean errors of the "Walking" of " $S_{1}$," " $S_{2}$," and " $S_{3}$ " that are calculated MTGP are 70.83, 60.41, and, 87.98, respectively. The results show that the mean absolute errors of " $S_{1}$," " $S_{2}$," and " $S_{3}$ " of four actions are smaller than TGP. Thus, MTGP is better than TGP, and MTGP can be employed to predict the arrival rates in this study.

In what follows, four experiments are displayed. In all numerical experiments, the minimization and maximization of the green time are $7 \mathrm{~s}$ and $50 \mathrm{~s}$, respectively; the yellow time $d_{y}=3 \mathrm{~s}$.

In the first, all arguments are given excluding the arrival rates. The cycles in the experiments are 100 and 200. Five sets of arrival rates predicted that the arrival rates of each inflow lane with the latest 20 phases are haphazardly generated and the values of the arrival rates are between 0 and 1 of each phase in all cycles. The optimal green times of 100 cycles and 200 cycles with different arrival rates that are gained by the MTGPLP approach are displayed in Figures 7 and 8, respectively. In Figures 7 and 8, dissimilar green times are arranged in different phases at the same cycle and also the diversity green times are determined in different cycles at the same phase with different arrival rates of the entrance lanes. Furthermore, these results demonstrate that different green times are programmed with different arrival rates and verify that model (11) is effective and the MTPGLP algorithm is powerful.

On the contrary, the total times of 100 cycles and 200 cycles with the arrival rates are demonstrated in Figures 9(a) and 9 (b), respectively. In addition, the signal plans are fixed in general. The constant cycle time are set $132 \mathrm{~s}$ (30 s/phase $\times 4$ phases $+3 \mathrm{~s} /$ phase $\times 4$ phases $=132 \mathrm{~s}$ ), $172 \mathrm{~s}$, and $212 \mathrm{~s}$ to be displayed in Figures 9(a) and 9(b). The results state that the cycles time are also different with the arrival rates estimated by the MTGP with 5 sets of latest 20 phases inflow lanes arrival rates and that the MTGPLP approach and system (11) are effective. In addition, in view of Figures 9(a) and 9(b), comparing the differences between the total time of each cycle of model (11) and the fixed plans, the model of this paper is more effective. Since the results are better displayed with different arrival rates, the total time of 100 cycles and 200 cycles is displayed in Figure 10(a). The results show that the total time of all cycles with different arrival rates is shorter than the sum of the constant time and model (11) and the MTGPLP algorithm are successful and valid.

In the second experiments, all arguments are prearranged excluding the departure rates. Five sets of departure rates at each inflow traffic lane are arbitrarily produced and the departure rates are in $[0,1]$ of each phase in all cycles. The optimal green times of 100 cycles and 200 cycles are obtained by the LP algorithm based on the predicted departure rates to be exhibited in Figures 11 and 12, respectively. From Figures 11 and 12, diverse green times are planned in different phases at the same cycle and also the diversity green times are determined in different cycles at the same phase with different departure rates of the outflow lanes. Thus, Figures 11 and 12 manifest that different green times are planned with different departure rates by MTGPLP and also authenticate that model (11) and MTPGLP algorithm are effective. In addition, the total time of each cycle with different departure rates and the constant cycle time are set $132 \mathrm{~s} \quad(30 \mathrm{~s} /$ phase $\times 4$ phases $+3 \mathrm{~s} /$ phase $\times 4$ phases $=$ $132 \mathrm{~s}), 172 \mathrm{~s}$, and $212 \mathrm{~s}$ are exhibited in Figure 13. The results demonstrate that the signal plans calculated by model (11) are more reasonable than the constant plans at the intersection. At last, since the results are better displayed with different departure rates, the total time of 100 cycles and 200 cycles with different departure rates are shown in Figure 10(b). The results also show that the model and the MTGPLP algorithm are effective.

In the third experiments, all arguments are prearranged excluding the weight factors. The five weight factors sets are randomly created and the weight factors' values range from 0 to 10. The green times are displayed in Figures 14 and 15, respectively. From Figures 14 and 15, diverse green times are planned in different phases at the same cycle and also the diversity green times are determined in different cycles at the same phase with different weight factors of the inflow lanes. Consequently, Figures 14 and 15 indicate that different green times are organized according to different weight factors. In addition, the total time of each cycle with different weight factors and the constant cycle time set $132 \mathrm{~s}$ (30 s/phase $\times 4$ phases $+3 \mathrm{~s} /$ phase $\times 4$ phases $=132 \mathrm{~s}), 172 \mathrm{~s}$, and $212 \mathrm{~s}$ are exhibited in Figure 16. The results demonstrate that the signal plans calculated by model (11) are more reasonable than the constant plans at the intersection. At last, since the results are better displayed with different weight factors, the total time of 100 cycles and 200 cycles with different weight factors are shown in Figure 10(b). The results also show that the model and the MTGPLP algorithm are effective.

Furthermore, the total times of random departure rates and stochastic weight factors are presented in Figures 13 and 16 , respectively. The results reveal that the cycle times are also different with the different inflow lanes' departure rates 

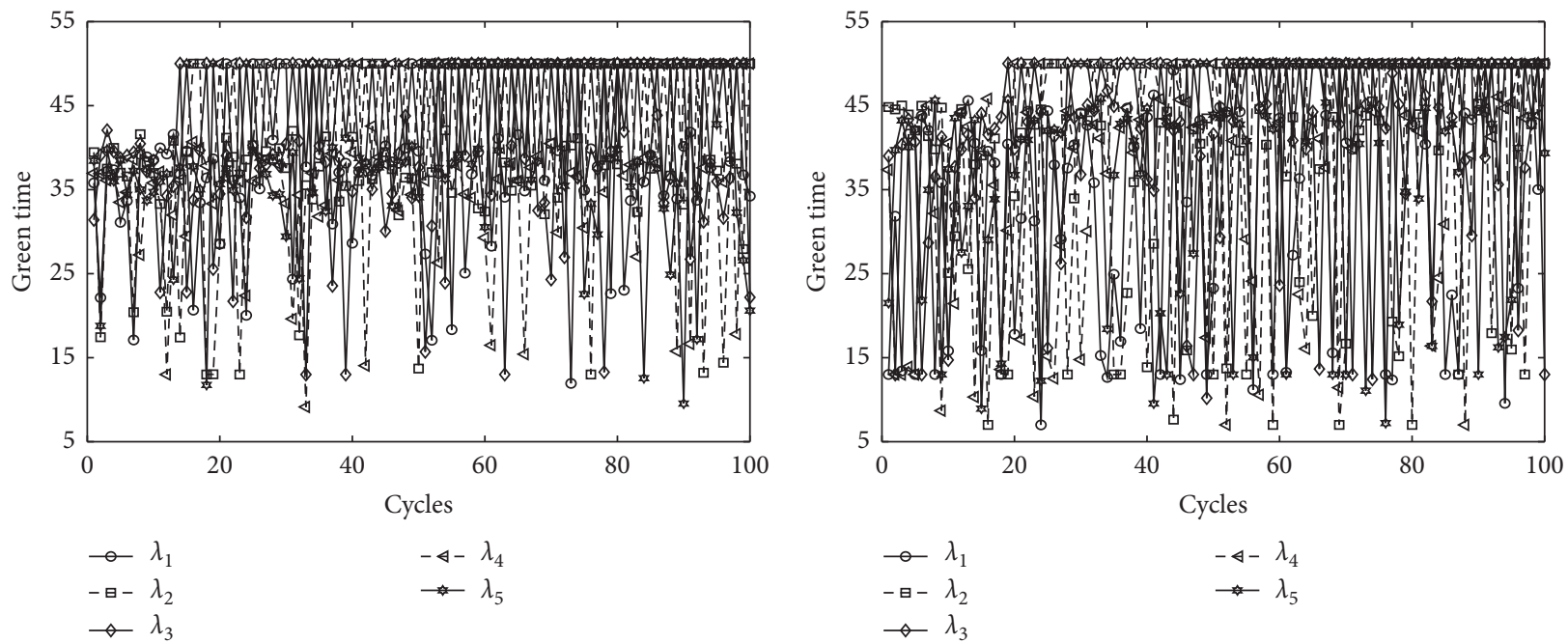

(a)

(b)

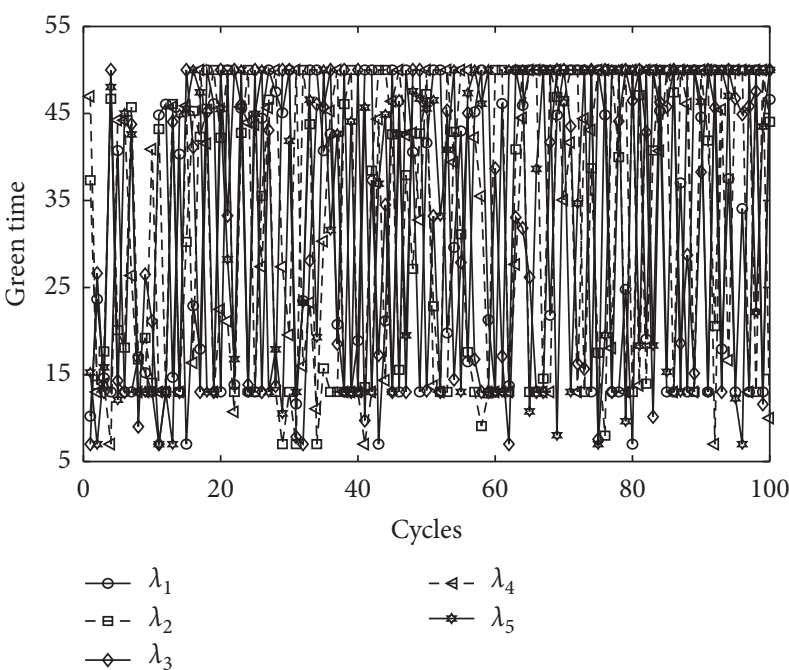

(c)
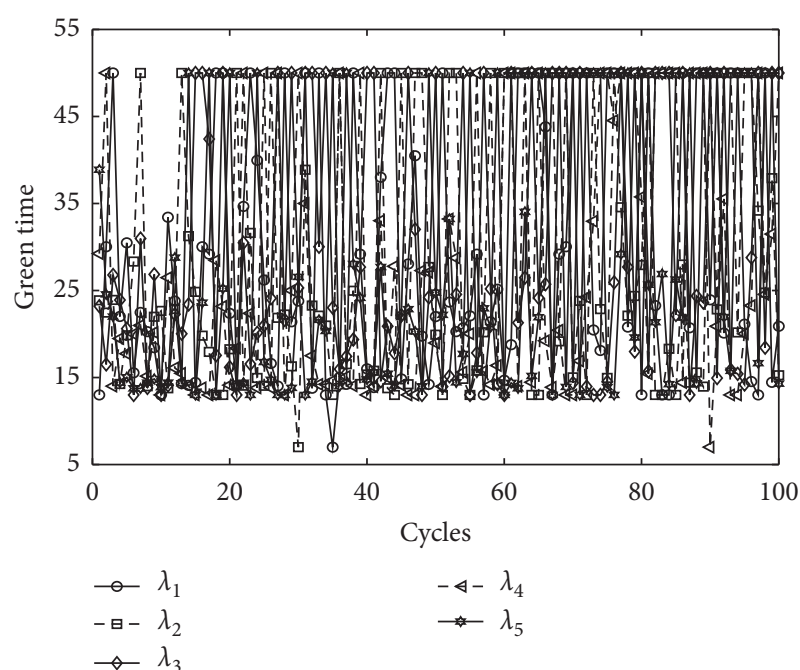

(d)

Figure 7: Green time of phases 1, 2, 3, and 4 of 100 cycles with 5 stochastic arrival rate sets. (a) Phase 1. (b) Phase 2. (c) Phase 3. (d) Phase 4.
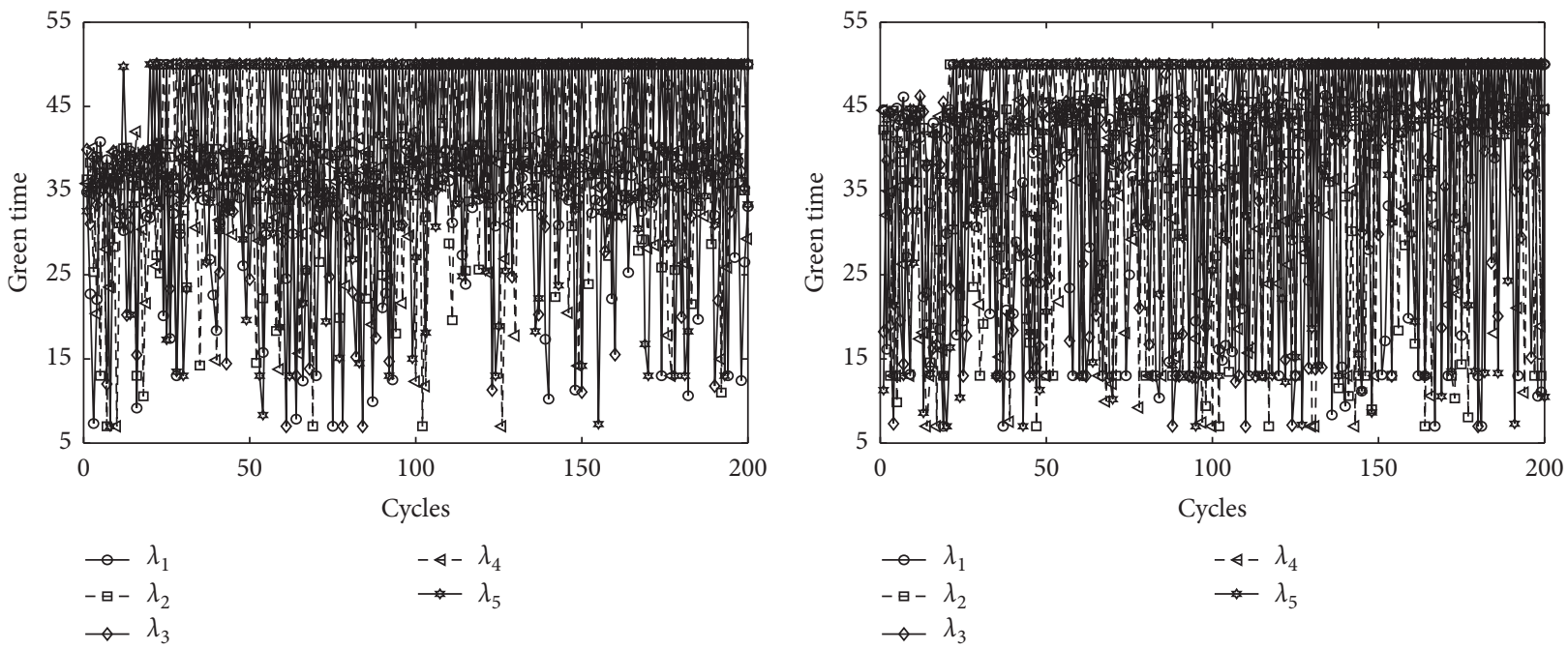

(a)

(b)

Figure 8: Continued. 


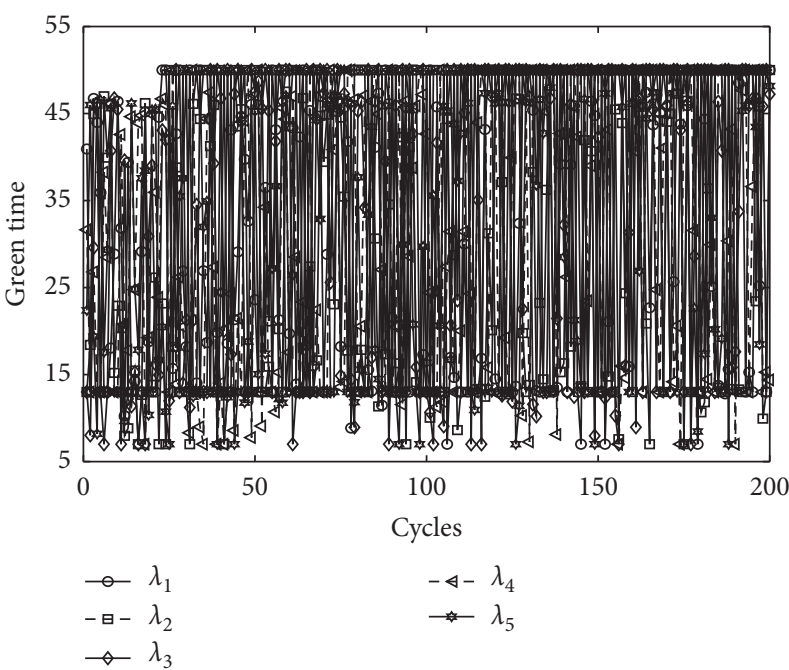

(c)

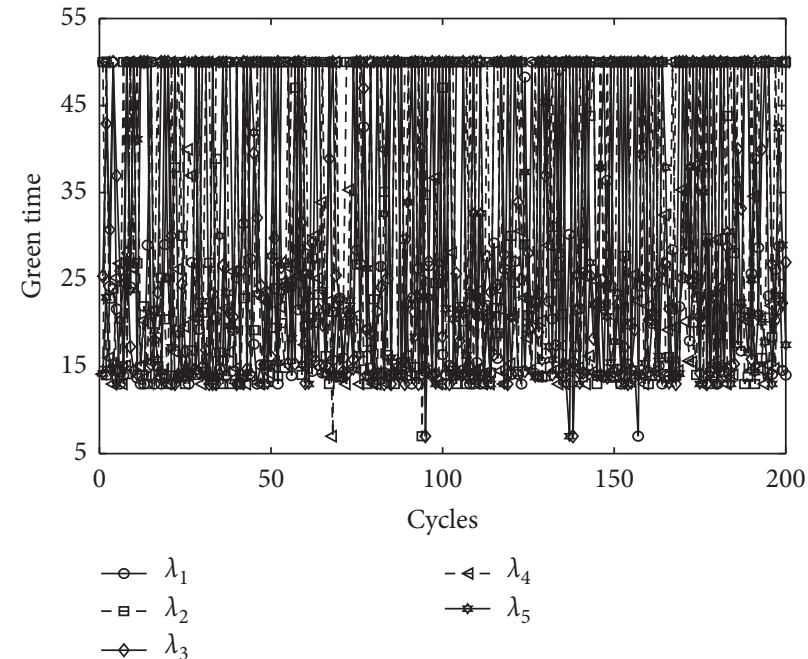

(d)

Figure 8: Green time of phases 1, 2, 3, and 4 of 200 cycles with 5 stochastic arrival rate sets. (a) Phase 1. (b) Phase 2. (c) Phase 3. (d) Phase 4.

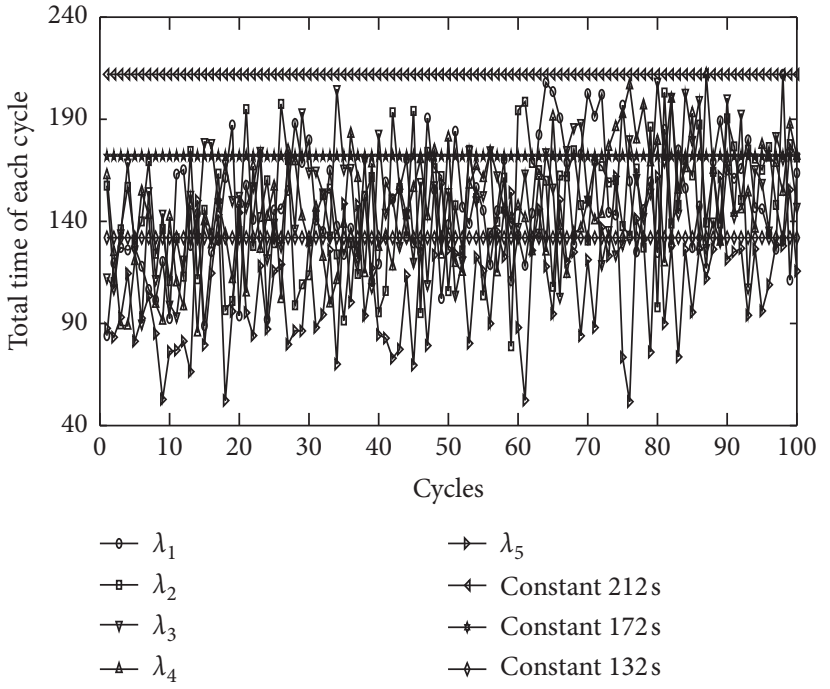

(a)

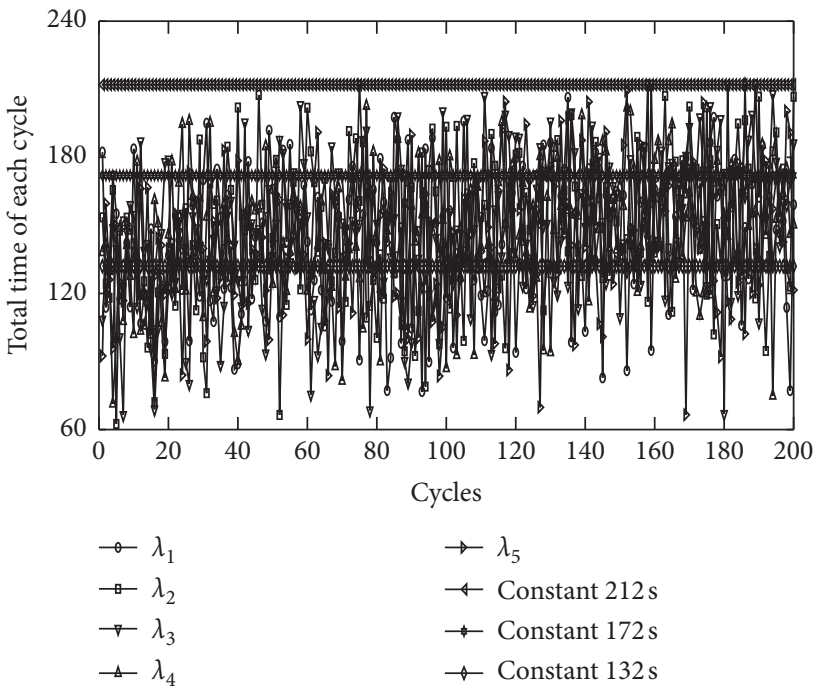

(b)

Figure 9: Cycles' time with 5 stochastic arrival rate sets. (a) 100 cycles. (b) 200 cycles.

and different weight factors of each inflow lane of every phase in each cycle. It also shows system (11) is effective.

In addition, the iterations of five sets of random arrival rates, departure rates, and weight factors of 100 cycles, 200 cycles, and 100 cycles are displayed in Figures 17(a)-17(c), respectively. The optimal values of different arrival rates, departure rates, and weight factors are obtained with a few steps of the algorithm cost. The results also demonstrate that the model is powerful and the MTGPLP algorithm is robust.

At last, combining all the number experiments, two other results can be obtained as follows: (1) different optimal green time with different number of cycles can be obtained; (2) system (11) is a powerful model and the algorithm is robust. 


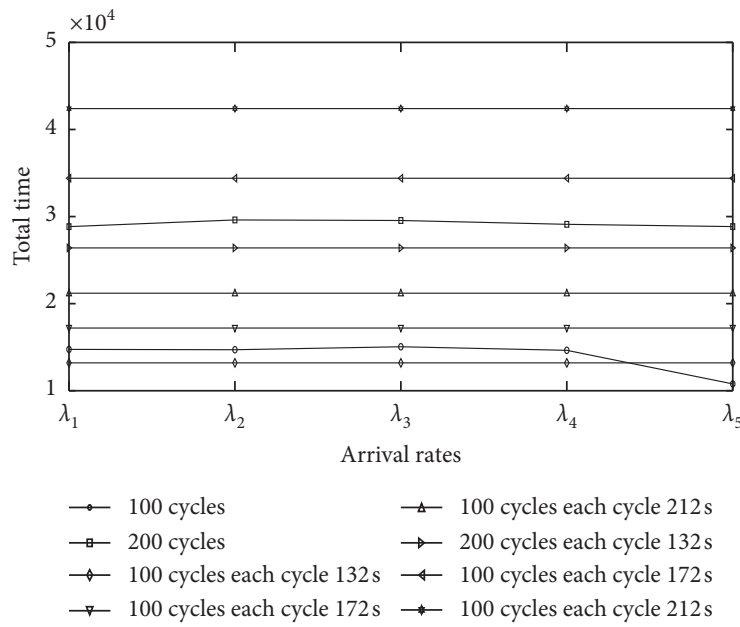

(a)
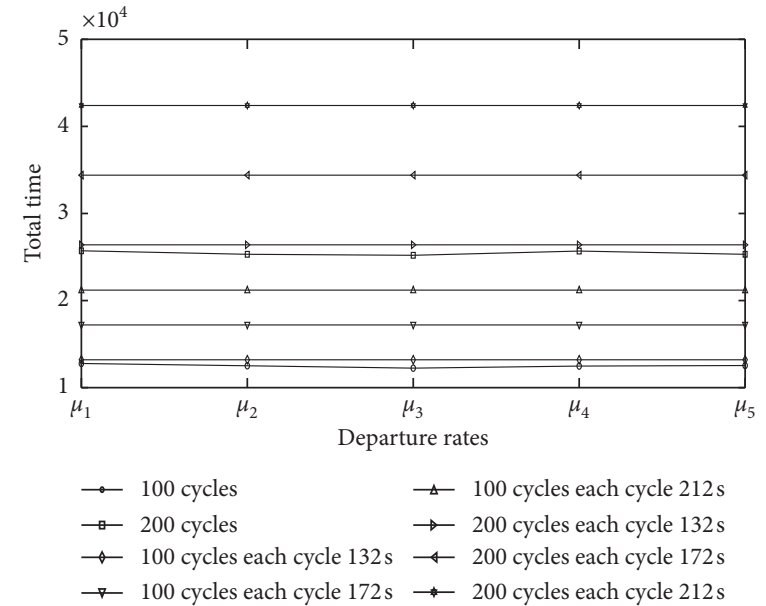

(b)

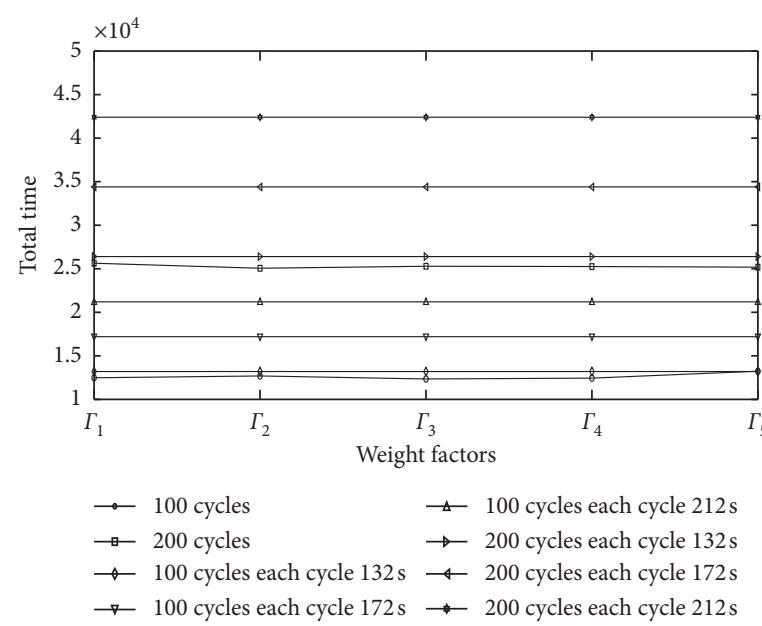

(c)

Figure 10: Total time of all cycles. (a) Five sets of randomly arrival rates (100 and 200 cycles). (b) Five sets of randomly departure rates (100 and 200 cycles). (c) Five sets of randomly weight factors (100 and 200 cycles).

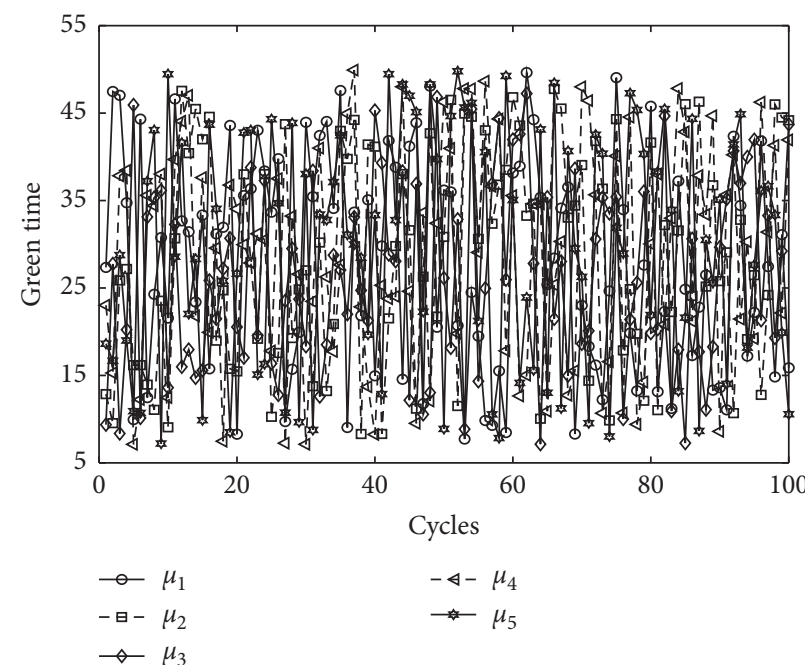

(a)

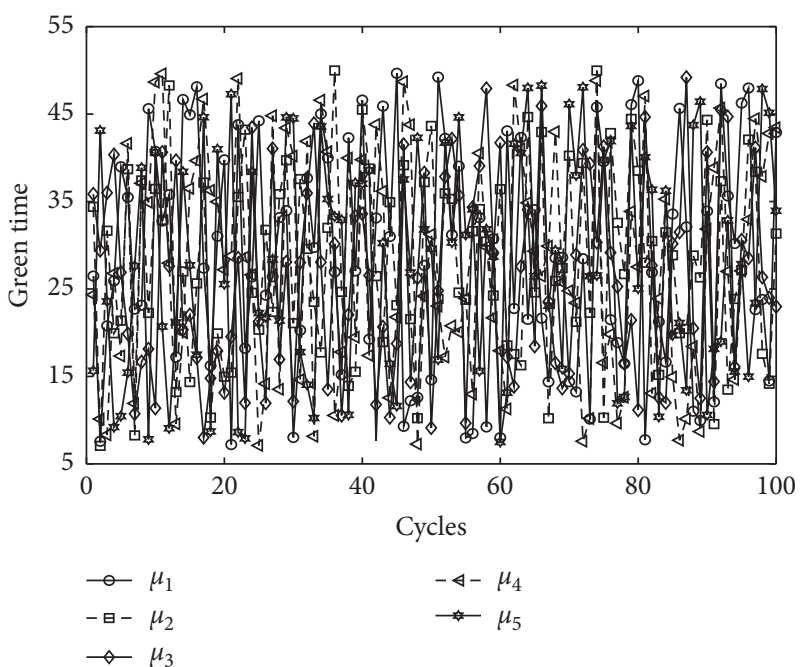

(b)

Figure 11: Continued. 


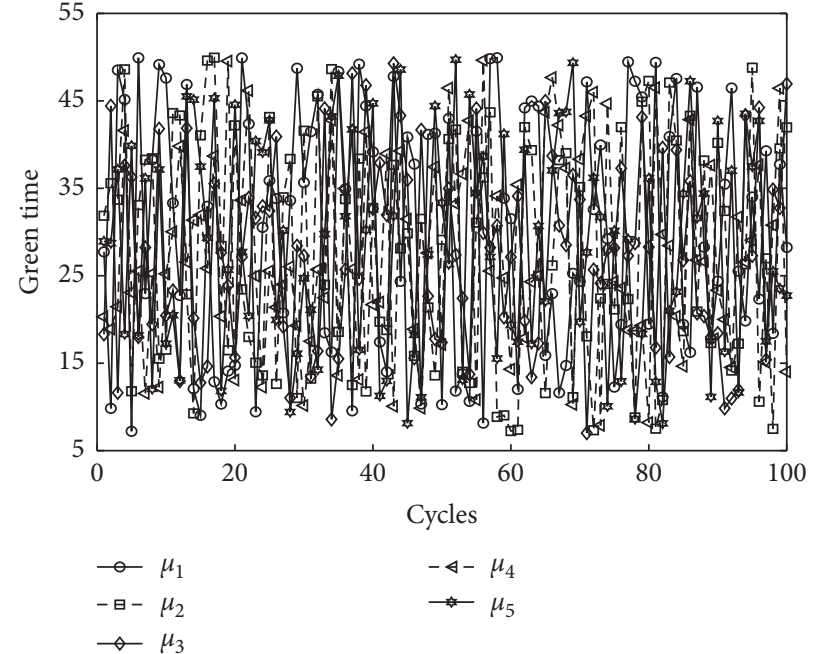

(c)

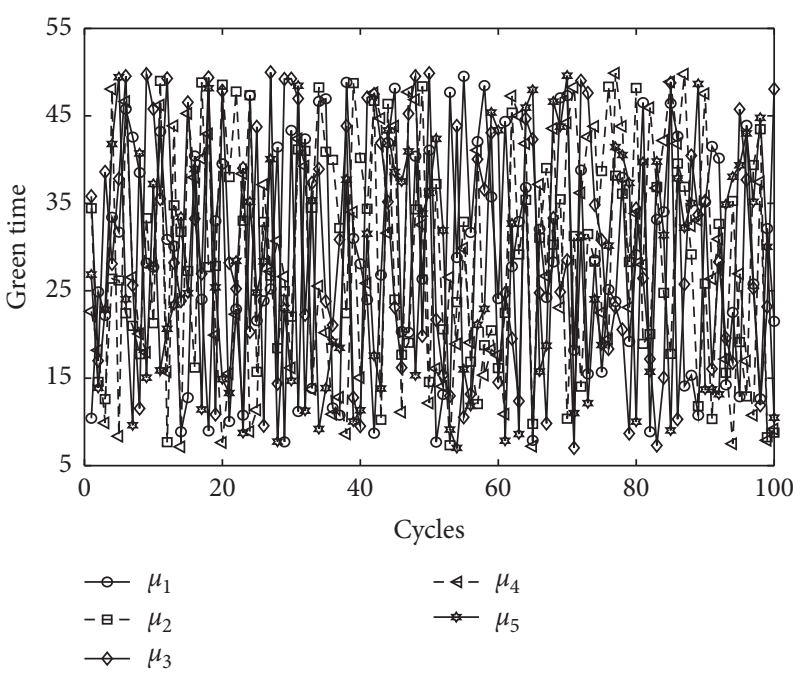

(d)

Figure 11: Green time of phases 1, 2, 3, and 4 of 100 cycles with 5 stochastic departure rate sets. (a) Phase 1. (b) Phase 2. (c) Phase 3. (d) Phase 4.
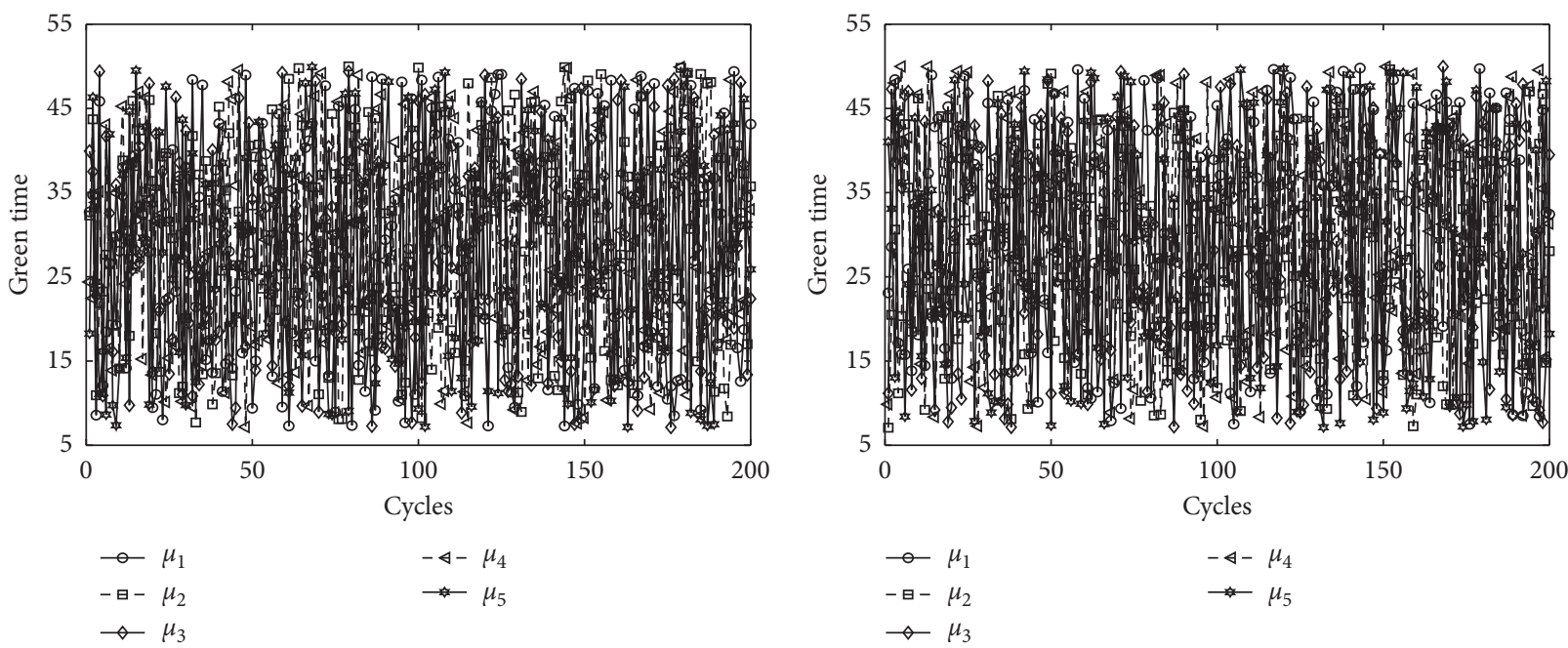

(a)

(b)
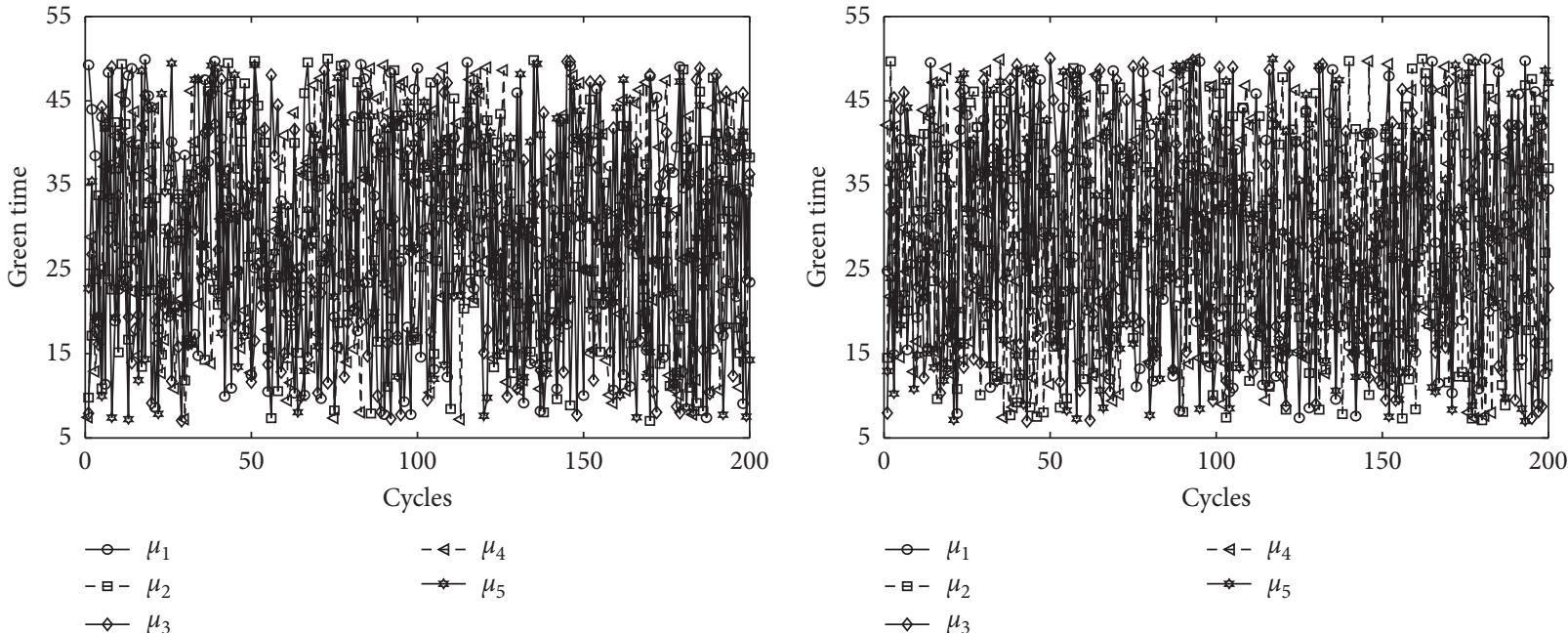

(c)

(d)

Figure 12: Green time of phases 1, 2, 3, and 4 of 200 cycles with 5 stochastic departure rate sets. (a) Phase 1. (b) Phase 2. (c) Phase 3. (d) Phase 4. 


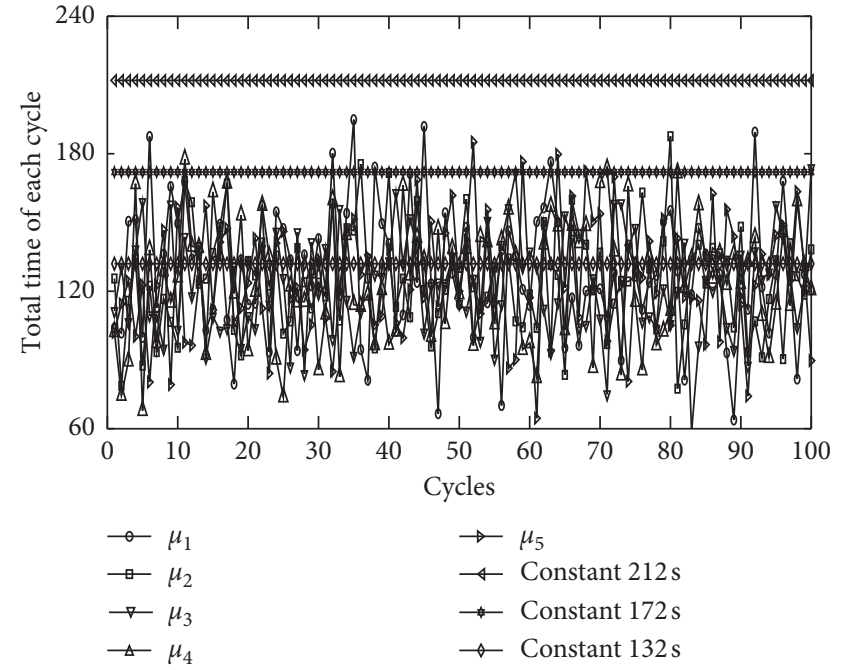

(a)

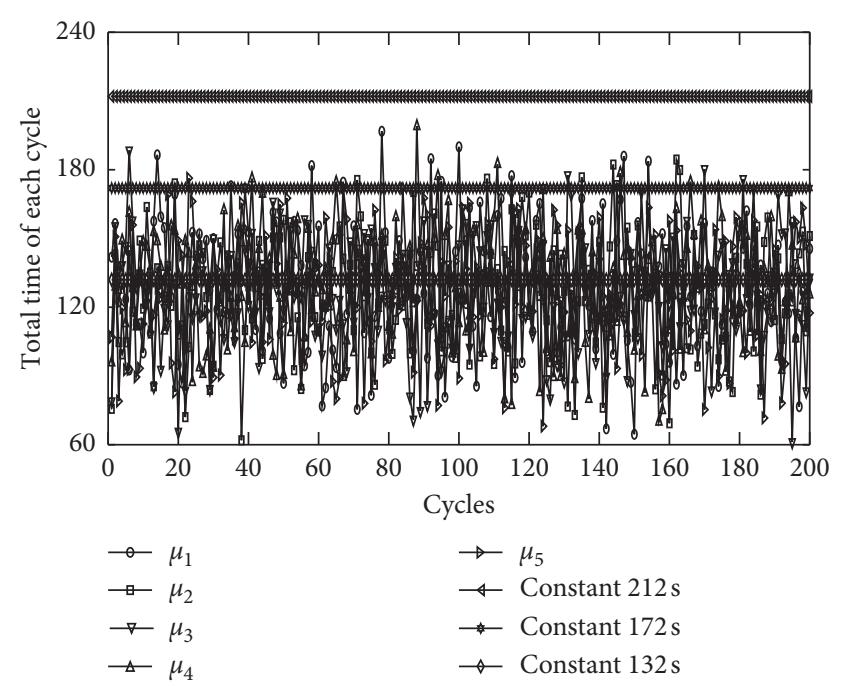

(b)

FIgURe 13: Cycles' time with 5 random departure rate sets. (a) 100 cycles with 5 sets' departure rates. (b) 200 cycles with 5 sets' departure rates.

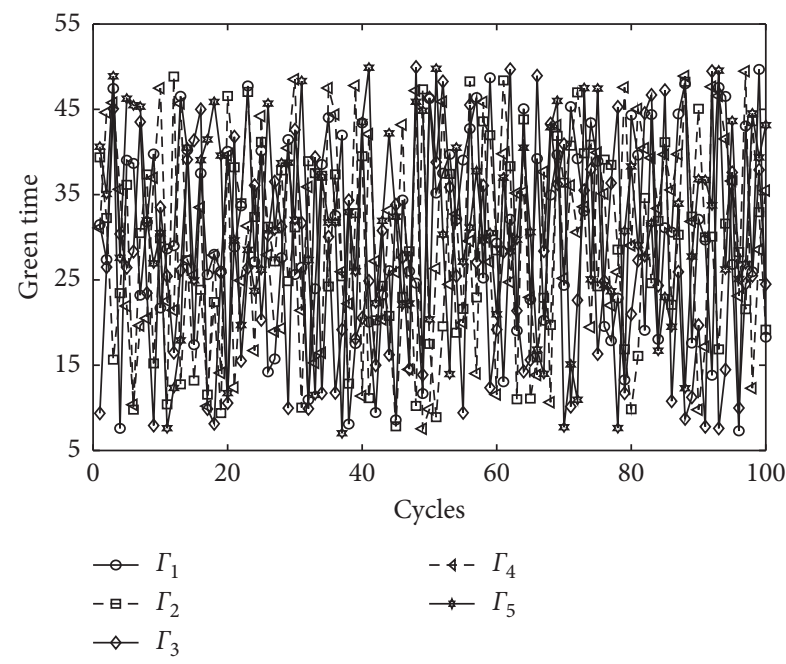

(a)

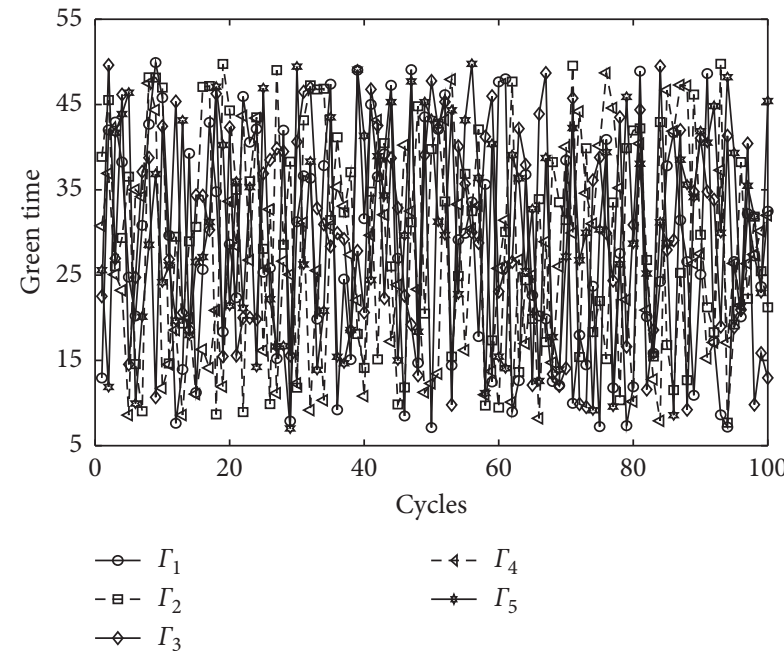

(c)

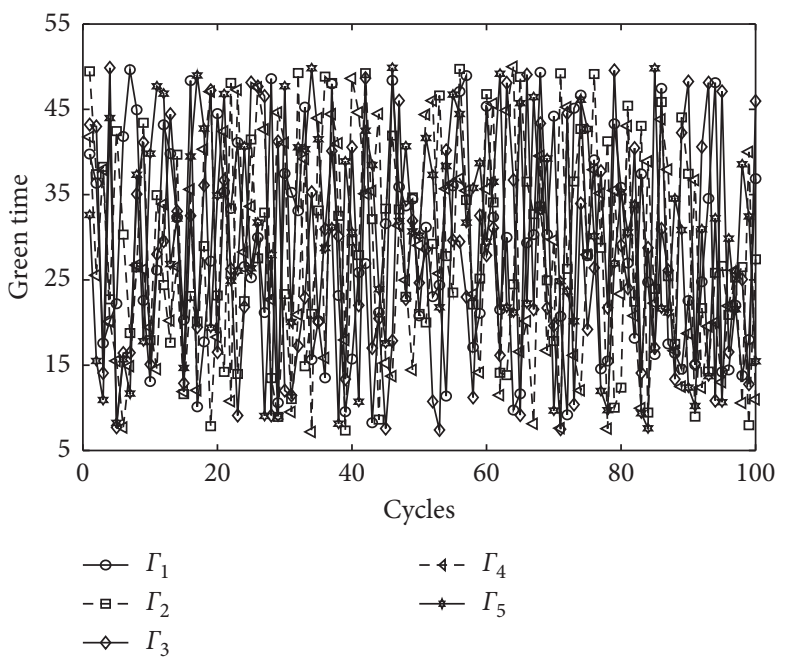

(b)

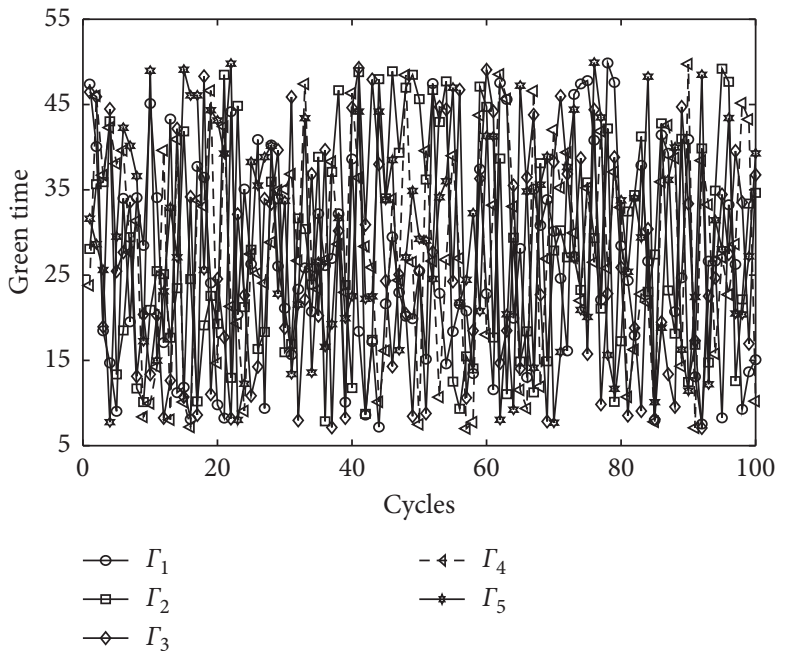

(d)

Figure 14: Green time of Phases 1, 2, 3, and 4 of 100 cycles with 5 stochastic weight factor sets. (a) Phase 1. (b) Phase 2. (c) Phase 3. (d) Phase 4. 

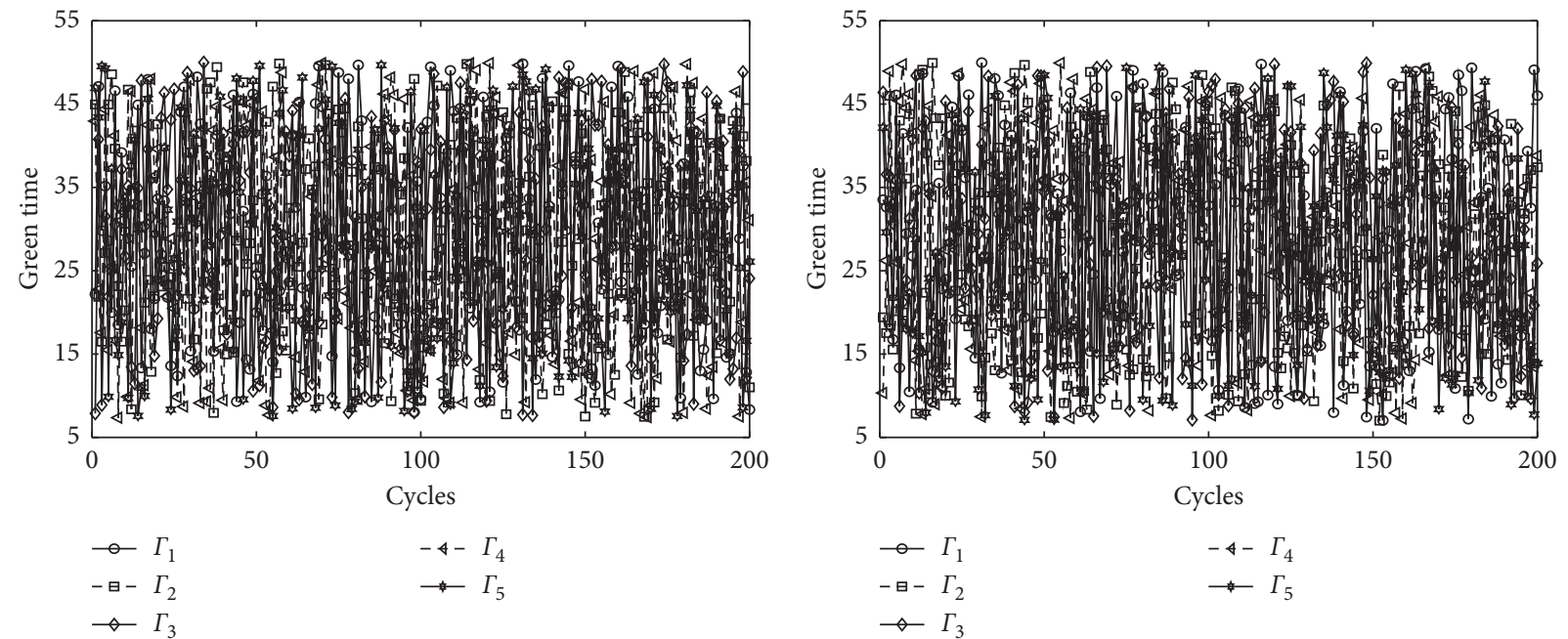

(a)

(b)

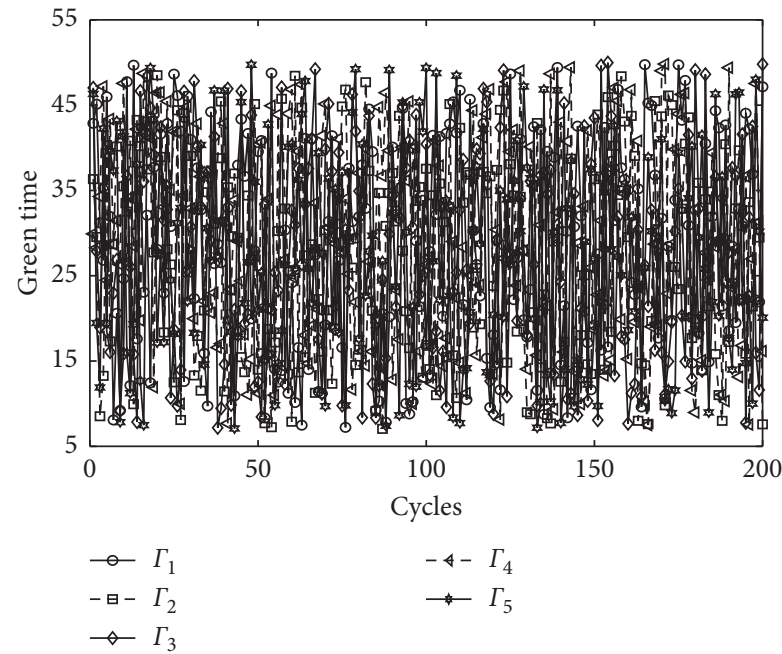

(c)

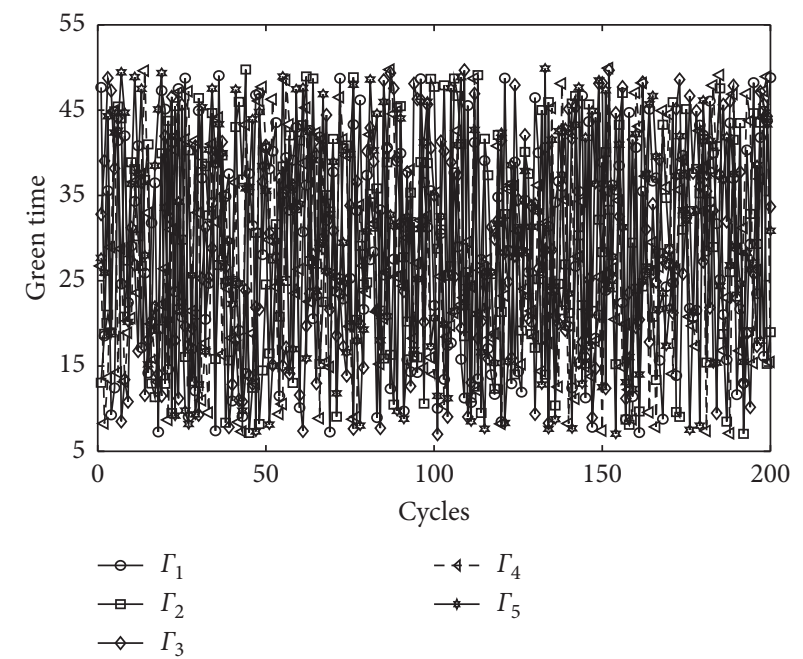

(d)

Figure 15: Green time of Phases 1, 2, 3, and 4 of 200 cycles with 5 stochastic weight factor sets. (a) Phase 1. (b) Phase 2. (c) Phase 3. (d) Phase 4.

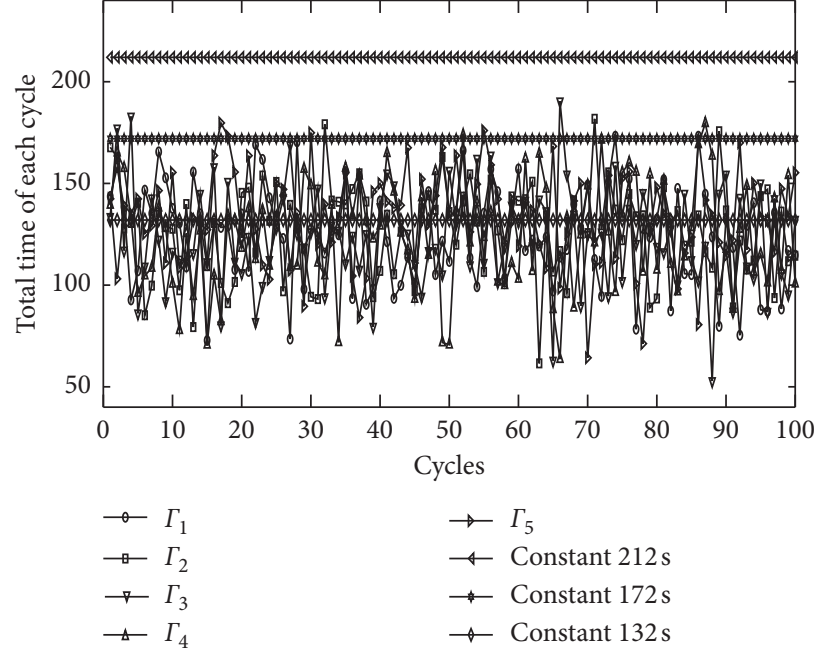

(a)

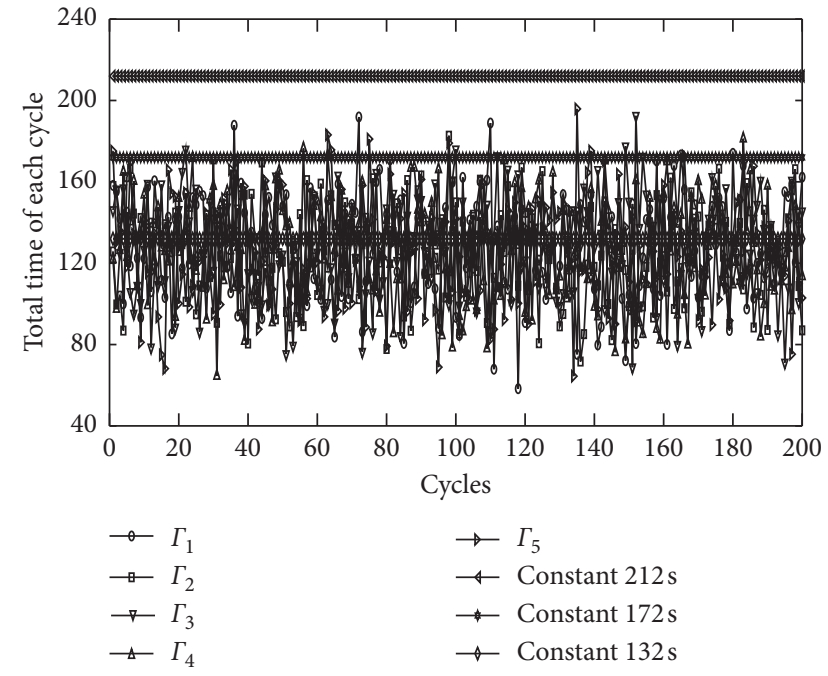

(b)

FIgURE 16: Cycles' time with 5 stochastic weight factor sets. (a) 100 cycles time. (b) 200 cycles time. 


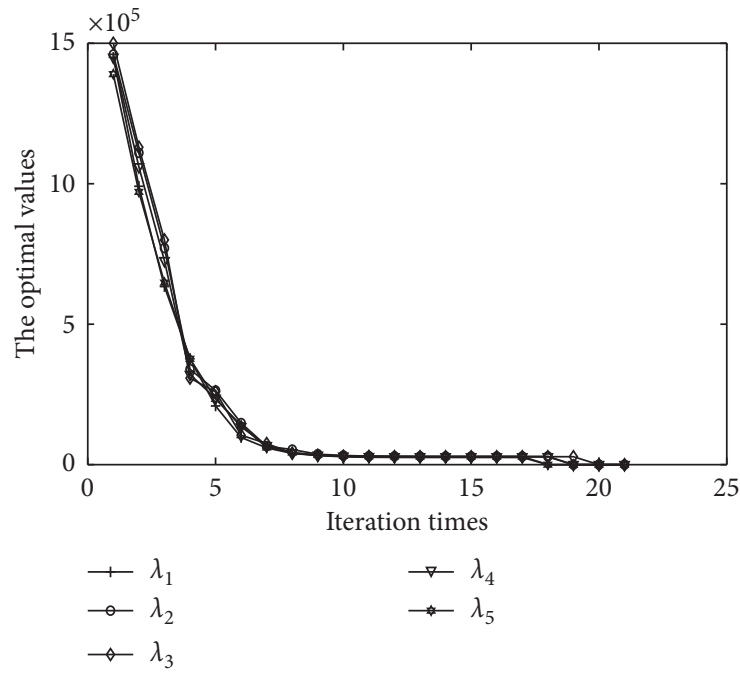

(a)

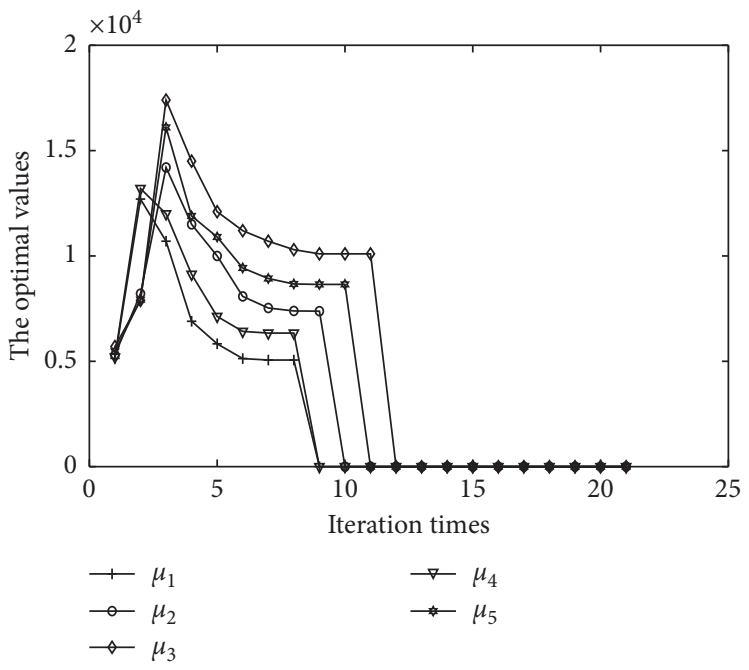

(b)

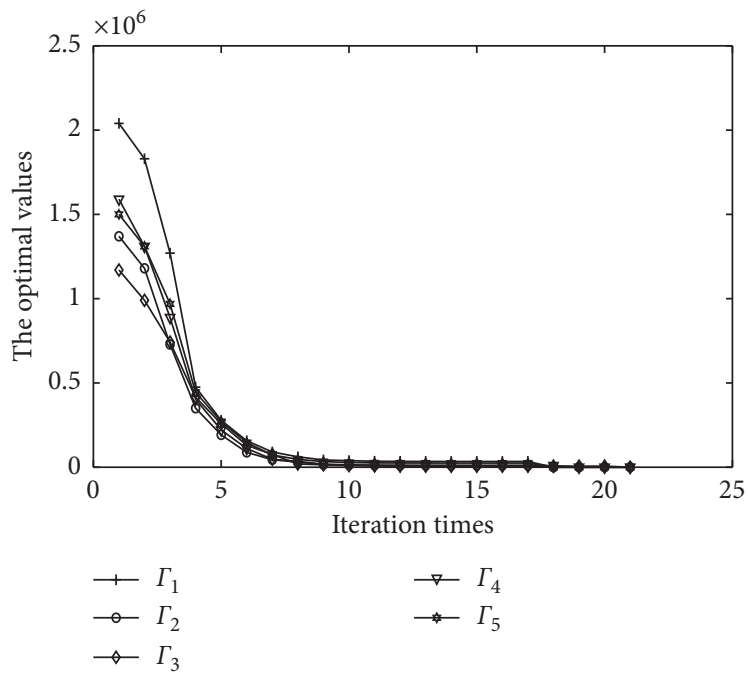

(c)

Figure 17: Convergence curves. (a) Five sets of random arrival rates (100 cycles). (b) Five sets of random arrival rates (200 cycles). (c) Five sets of random weight factors (100 cycles).

\section{Conclusions}

In general, it is difficult to determine the arrival rates at the intersection and the "max" operator is contained in the models. In the paper, the "max" operator is reformulated some linear inequalities. Thus, we introduce a novel combining method to solve the traffic signal time problem for controlling the traffic light of a single junction. In the method, a modified multioutput algorithm (MTGP), which is better than TGP that is proved by the first number experiment, is presented which is utilized to predict the arrival rates during phases of each cycle. Thus, the arrival rates of the entrance lanes are solved. Then, the intelligent framework introduced is realized. In view of the numerical experiments by the MTGPLP method, some conclusions can be drawn as follows. (1) The system of this paper is successful, that is to say, diverse arrival rates, different departure rates, or different weight factors can lead to different optimal green time by the system. (2) A novel intelligent framework for the traffic signal control problem is stated. (3) In the intelligent framework, for the sake of achieving automatic of traffic signal control with optimal of each phase based on different arrival rates, the MTGPLP algorithm is contained. (4) According to the numerical experiments, the model and the MTGPLP approach are effective and validity. On the contrary, since the arrival rates of multi-intersection are interacted, MTGPLP algorithm cannot extend to the situation of multiintersection. Thus, some more complicated situations (e.g., multi-intersection, vehicles, and pedestrians) can be investigated in the future.

\section{Data Availability}

The datasets of the arrival rates, departure rates, and weight factors are stochastically generated in the manuscript. The datasets of the first experiment are obtained in [69]. 


\section{Conflicts of Interest}

The authors declare that they have no conflicts of interest.

\section{Acknowledgments}

This work was supported in part by the NSFC (no. 11901320).

\section{References}

[1] P. Koonce and L. Rodegerdts, Traffic Signal Timing Manual, Federal Highway Administration, Washington, DC, USA, 2008.

[2] F. V. Webster, Traffic Signal Settings, Her Majesty's Stationery Office, London, UK, 1958.

[3] D. I. Robertson, "Transyt, a traffic network study tool," Technical Report TRRL-LR-253, Transport and Road Research Laboratory, Crowthorne, UK, 1969.

[4] G. E. Cantarella and G. Improta, "Capacity factor or cycle time optimization for signalized junctions: a graph theory approach," Transportation Research Part B: Methodological, vol. 22, no. 1, pp. 1-23, 1988.

[5] S. Reljic, "Multicriteria optimal control in a network of signalised intersections," in Proceedings of the Internaional Symposium on Transportation and Traffic Theory, pp. 615-627, Lyon, France, July 1996.

[6] S. C. Wong, "Group-based optimisation of signal timings using parallel computing," Transportation Research Part C: Emerging Technologies, vol. 5, no. 2, pp. 123-139, 1997.

[7] C. K. Wong and S. C. Wong, "A lane-based optimization method for minimizing delay at isolated signal-controlled junctions," Journal of Mathematical Modelling and Algorithms, vol. 2, no. 4, pp. 379-406, 2003.

[8] P. R. Lowrie, "The Sydney coordinated adaptive traffic systemprinciples, methodology, algorithms," in Proceedings of the International Conference on Road Traffic Signalling, pp. 67-70, London, UK, March 1982.

[9] D. Bretherton and G. T. Bowen, "Recent enhancements to SCOOT-SCOOT version 2.4," in Proceedings of the $3 \mathrm{rd}$ Intrenational Conference on Road Traffic Control, pp. 95-98, London, UK, May 1990.

[10] D. Bretherton, "SCOOT MC3 and current developments," in Proceedings of the Transportation Research Board 86th Annual Meeting (CD-ROM), Washington, DC, USA, January 2007.

[11] S. Sharma and M. R. Gidde, "Estimated outcome of application of split cycle offset optimization technique (SCOOT) and reduction of frequency of buses," International Journal of Engineering Research and Management Technology, vol. 1, no. 5, pp. 82-90, 2014.

[12] W.-M. Wey, "Model formulation and solution algorithm of traffic signal control in an urban network," Computers, Environment and Urban Systems, vol. 24, no. 4, pp. 355-378, 2000.

[13] J. B. Clempner and A. S. Poznyak, "Modeling the multi-traffic signal-control synchronization: a Markov chains game theory approach," Engineering Applications of Artificial Intelligence, vol. 43, pp. 147-156, 2015.

[14] K. L. Head, P. B. Mirchandani, and D. Sheppard, "Hierarchical framework for real-time traffic control," Transportation Research Record, vol. 1360, pp. 82-88, 1992.

[15] P. Mirchandani and L. Head, "A real-time traffic signal control system: architecture, algorithms, and analysis,"
Transportation Research Part C: Emerging Technologies, vol. 9, no. 6, pp. 415-432, 2001.

[16] M. Dotoli, M. P. Fanti, and C. Meloni, "A signal timing plan formulation for urban traffic control," Control Engineering Practice, vol. 14, no. 11, pp. 1297-1311, 2006.

[17] J.-D. Schmöcker, S. Ahuja, and M. G. H. Bell, "Multi-objective signal control of urban junctions - framework and a London case study," Transportation Research Part C: Emerging Technologies, vol. 16, no. 4, pp. 454-470, 2008.

[18] C. K. Wong and S. C. Wong, "Lane-based optimization of signal timings for isolated junctions," Transportation Research Part B: Methodological, vol. 37, no. 1, pp. 63-84, 2003.

[19] C. K. Wong, S. C. Wong, and C. O. Tong, "A lane-based optimization method for the multi-period analysis of isolated signal-controlled junctions," Transportmetrica, vol. 2, no. 1, pp. 53-85, 2006.

[20] J. Zhang, F.-Y. Wang, K. Wang, W.-H. Lin, X. Xu, and C. Chen, "Data-driven intelligent transportation systems: a survey," IEEE Transactions on Intelligent Transportation Systems, vol. 12, no. 4, pp. 1624-1639, 2011.

[21] S. Kachroudi and N. Bhouri, "A multimodal traffic responsive strategy using particle swarm optimization," IFAC Proceedings Volumes, vol. 42, no. 15, pp. 531-537, 2009.

[22] C. Cai, C. K. Wong, and B. G. Heydecker, "Adaptive traffic signal control using approximate dynamic programming," Transportation Research Part C: Emerging Technologies, vol. 17 , no. 5, pp. 456-474, 2009.

[23] M. A. Khamis and W. Gomaa, "Adaptive multi-objective reinforcement learning with hybrid exploration for traffic signal control based on cooperative multi-agent framework," Engineering Applications of Artificial Intelligence, vol. 29, pp. 134-151, 2014.

[24] Y. Dujardin, D. Vanderpooten, and F. Boillot, "A multi-objective interactive system for adaptive traffic control," European Journal of Operational Research, vol. 244, no. 2, pp. 601-610, 2015.

[25] J. Garcia-Nieto, A. C. Olivera, and E. Alba, "Optimal cycle program of traffic lights with particle swarm optimization," IEEE Transactions on Evolutionary Computation, vol. 17, no. 6, pp. 823-839, 2013.

[26] L. Li, K. Yang, Z. Li, and Z. Zhang, "The optimality condition of the multiple-cycle smoothed curve signal timing model," Transportation Research Part C: Emerging Technologies, vol. 27, pp. 46-57, 2013.

[27] P. Coll, P. Factorovich, I. Loiseau, and R. Gómez, “A linear programming approach for adaptive synchronization of traffic signals," International Transactions in Operational Research, vol. 20, no. 5, pp. 667-679, 2013.

[28] T. L. Friesz, K. Han, P. A. Neto, A. Meimand, and T. Yao, "Dynamic user equilibrium based on a hydrodynamic model," Transportation Research Part B: Methodological, vol. 47, pp. 102-126, 2013.

[29] R. Liu and M. Smith, "Route choice and traffic signal control: a study of the stability and instability of a new dynamical model of route choice and traffic signal control," Transportation Research Part B: Methodological, vol. 77, pp. 123-145, 2015.

[30] K. Han, V. V. Gayah, B. Piccoli, T. L. Friesz, and T. Yao, "On the continuum approximation of the on-and-off signal control on dynamic traffic networks," Transportation Research Part B: Methodological, vol. 61, pp. 73-97, 2014.

[31] K. Han and V. V. Gayah, "Continuum signalized junction model for dynamic traffic networks: offset, spillback, and multiple signal phases," Transportation Research Part B: Methodological, vol. 77, pp. 213-239, 2015. 
[32] K. Han, H. Liu, V. V. Gayah, T. L. Friesz, and T. Yao, “A robust optimization approach for dynamic traffic signal control with emission considerations," Transportation Research Part C: Emerging Technologies, vol. 70, pp. 3-26, 2016.

[33] Y.-S. Qian, X. Feng, and J.-W. Zeng, "A cellular automata traffic flow model for three-phase theory," Physica A: Statistical Mechanics and Its Applications, vol. 479, pp. 509-526, 2017a.

[34] Y. Qian, J. Zeng, N. Wang, J. Zhang, and B. Wang, "A traffic flow model considering influence of car-following and its echo characteristics," Nonlinear Dynamics, vol. 89, no. 2, pp. 1099-1109, 2017b.

[35] C. Yu, W. Ma, K. Han, and X. Yang, "Optimization of vehicle and pedestrian signals at isolated intersections," Transportation Research Part B: Methodological, vol. 98, pp. 135153, 2017a.

[36] D. Manolis, I. Papamichail, E. B. Kosmatopoulos, and M. Papageorgiou, "Automated tuning of ITS management and control systems: results from real-life experiments," Transportation Research Part C: Emerging Technologies, vol. 66, pp. 119-135, 2016.

[37] S. Choi, B. B. Park, J. Lee, H. Lee, and S. H. Son, "Field implementation feasibility study of cumulative travel-time responsive (CTR) traffic signal control algorithm," Journal of Advanced Transportation, vol. 50, no. 8, pp. 2226-2238, 2016.

[38] B. Abdulhai, R. Pringle, and G. J. Karakoulas, "Reinforcement learning for true adaptive traffic signal control," Journal of Transportation Engineering, vol. 129, no. 3, pp. 278-285, 2003.

[39] M. Abdoos, N. Mozayani, and A. L. Bazzan, "Traffic light control in non-stationary environments based on multi agent Q-learning," in Proceedings of the 2011 14th International IEEE Conference on Intelligent Transportation Systems (ITSC), pp. 1580-1585, Washington, DC, USA, October 2011.

[40] A. Kouvelas, K. Aboudolas, E. B. Kosmatopoulos, and M. Papageorgiou, "Adaptive performance optimization for large-scale traffic control systems," IEEE Transactions on Intelligent Transportation Systems, vol. 12, no. 4, pp. 14341445, 2011.

[41] S. El-Tantawy, B. Abdulhai, and H. Abdelgawad, "Multiagent reinforcement learning for integrated network of adaptive traffic signal controllers (MARLIN-ATSC): methodology and large-scale application on downtown Toronto," IEEE Transactions on Intelligent Transportation Systems, vol. 14, no. 3, pp. 1140-1150, 2013.

[42] S. Araghi, A. Khosravi, M. Johnstone, and D. Creighton, "Qlearning method for controlling traffic signal phase time in a single intersection," in Proceedings of the 16th International IEEE Conference on Intelligent Transportation Systems (ITSC 2013), pp. 1261-1265, The Hague, Netherlands, October 2013.

[43] S. Jadhav, "Traffic signal management using machine learning algorithm," International Journal of Engineering and Technical Research, vol. 9, no. 6, pp. 384-387, 2020.

[44] D. Srinivasan, M. C. Choy, and R. L. Cheu, "Neural networks for real-time traffic signal control," IEEE Transactions on Intelligent Transportation Systems, vol. 7, no. 3, pp. 261-272, 2006.

[45] M. Kaedi, N. Movahhedinia, and K. Jamshidi, “Traffic signal timing using two-dimensional correlation, neuro-fuzzy and queuing based neural networks," Neural Computing and Applications, vol. 17, no. 2, pp. 193-200, 2008.

[46] F. M. Sisido, J. H. H. Goya, G. S. Bastos, and A. W. Li, "Traffic signs recognition system with convolution neural networks," in Proccedings of the Latin American Robotic Symposium, 2018 Brazilian Symposium on Robotics and 2018 Workshop on
Robotics in Education, pp. 339-344, Joao Pessoa, Brazil, November 2018.

[47] Y. K. Ki, N. W. Heo, J. W. Choi, G. H. Ahn, and K. S. Park, "An incident detection algorithm using artificial neural networks and traffic information," in Proceedings of the 2018 Cybernetics and Informatics, pp. 1-5, Lazy pod Makytou, Slovakia, February 2018.

[48] C.-H. Wan and M.-C. Hwang, "Value-based deep reinforcement learning for adaptive isolated intersection signal control," IET Intelligent Transport Systems, vol. 12, no. 9, pp. 1005-1010, 2018.

[49] S. Wang, X. Xie, K. Huang, J. Zeng, and Z. Cai, "Deep reinforcement learning-based traffic signal control using highresolution event-based data," Entropy, vol. 21, no. 8, pp. 744-16, 2019.

[50] K. L. Tan, S. Poddar, A. Sharma, and S. Sarkar, "Deep reinforcement learning for adaptive traffic signal control," Proceedings of the ASME 2019 Dynamic Systems and Control Conference, vol. 3, Park City, Utah, October 2019.

[51] M. Gregurić, M. Vujić, C. Alexopoulos, and M. Miletić, "Application of deep reinforcement learning in traffic signal control: an overview and impact of open traffic data," Applied Sciences, vol. 10, no. 11, pp. 4011-25, 2020.

[52] Y. K. Chin, N. Bolong, S. S. Yang, and K. T. K. Teo, "Exploring Q-learning optimization in traffic signal timing plan management," International Journal of Simulation, Systems, Science and Technology, vol. 12, no. 3, pp. 29-35, 2011.

[53] J. Zeng, J. Hu, and Y. Zhang, "Adaptive traffic signal control with deep recurrent Q-learning," in Proceedings of the IEEE Intelligent Vehicles Symposium, pp. 1215-1220, Changshu, China, June 2018.

[54] M. J. Moghaddam, M. Hosseini, and R. Safabakhsh, "Fuzzy Q-learning traffic light control based on traffic flow and pedestrian number estimated from visual information," Journal of Computer and Knowledge Engineering, vol. 2, no. 1, pp. 1-10, 2019.

[55] X. Liang, X. Du, G. Wang, and Z. Han, “A deep reinforcement learning network for traffic light cycle control," IEEE Transactions on Vehicular Technology, vol. 68, no. 2, pp. 1243-1253, 2019.

[56] W. Genders and S. Razavi, “Asynchronous n-step Q-learning adaptive traffic signal control," Journal of Intelligent Transportation Systems, vol. 23, no. 4, pp. 319-331, 2019.

[57] I. M. Ribeiro and M. L. Simões, "Optimal cycle for a signalized intersection using global optimization and complementarity," TOP, vol. 20, no. 3, pp. 777-790, 2012.

[58] M. L. Simões and I. M. Ribeiro, "Global optimization and complementarity for solving a semi- actuated traffic control problem," Procedia-Social and Behavioral Sciences, vol. 20, pp. 390-397, 2011.

[59] M. L. Simões and I. M. Ribeiro, "Optimal signal timing signalized intersection by global optimization," in Proceedings of the 1st International Conference on Engineering and Applied Sciences Optimization, pp. 664-679, Kos, Greece, June 2014.

[60] I. M. Ribeiro and M. d. L. d. O. Simões, "The fully actuated traffic control problem solved by global optimization and complementarity," Engineering Optimization, vol. 48, no. 2, pp. 199-212, 2016.

[61] H. Liu, K. N. Balke, and W.-H. Lin, “A reverse causal-effect modeling approach for signal control of an oversaturated intersection," Transportation Research Part C: Emerging Technologies, vol. 16, no. 6, pp. 742-754, 2008.

[62] Y. Li, L. Yu, S. Tao, and K. Chen, "Multi-objective optimization of traffic signal timing for oversaturated intersection," 
Mathematical Problems in Engineering, vol. 2013, Article ID 182643, 9 pages, 2013.

[63] L. Zhao, X. Peng, L. Li, and Z. Li, "A fast signal timing algorithm for individual oversaturated intersections," IEEE Transactions on Intelligent Transportation Systems, vol. 12, no. 1, pp. 280-283, 2010.

[64] Y. Tong, L. Zhao, L. Li, and Y. Zhang, "Stochastic programming model for oversaturated intersection signal timing," Transportation Research Part C: Emerging Technologies, vol. 58, pp. 474-486, 2015.

[65] Y.-Y. Zou and Y. Hu, "An approach of traffic signal control based on NLRSQP algorithm," Modern Physics Letters B, vol. 31, no. 31, Article ID 1750293, 2017.

[66] Y.-Y. Zou, X.-G. Xu, and G.-H. Lin, "Adaptive multi-objective traffic signal control using NLRMNSGA-II algorithm," Canadian Journal of Civil Engineering, vol. 45, no. 11, pp. 973985, 2018.

[67] L. Bo and C. Sminchisescu, "Twin Gaussian processes for structured prediction," International Journal of Computer Vision, vol. 87, no. 1-2, pp. 28-52, 2010.

[68] B. De Schutter and B. De Moor, "Optimal traffic light control for a single intersection," European Journal of Control, vol. 4, no. 3, pp. 260-276, 1998.

[69] L. Sigal and M. Black, "HumanEva: synchronized video and motion capture dataset for evaluation of articulated human motion," International Journal of Computer Vision, vol. 87, p. 4, 2006 . 